\title{
The role and value of negative emissions technologies in , decarbonising the UK energy system \\ 2
}

\author{
H. A. Daggash ${ }^{1,2,3}$, C. F. Heuberger ${ }^{2,3}$, and N. Mac Dowell ${ }^{* 2,3}$ \\ ${ }^{1}$ Grantham Institute for Climate Change and the Environment, Imperial College $\quad 4$ \\ London, Exhibition Road, London, UK \\ ${ }^{2}$ Centre for Environmental Policy, Imperial College London, Exhibition Road, 6 \\ London, UK \\ ${ }^{3}$ Centre for Process Systems Engineering, Imperial College London, Exhibition $\quad 8$ \\ Road, London, UK
}

\begin{abstract}
The UK is committed to the Paris Agreement and has a legally-binding target to reduce 11 economy-wide greenhouse gas emissions by $80 \%$ relative to 1990 levels by 2050 . Meeting 12 these targets would require deep decarbonisation, including the deployment of negative emissions 13 technologies. This study, via a power supply capacity expansion model, investigates the potential 14 role of bio-energy with carbon capture and storage (BECCS) and direct air capture and storage 15 (DACS) in meeting the UK's emissions reduction targets. We show that to achieve power sector 16 decarbonisation, a system dominated by firm and dispatchable low-carbon generators with BECCS 17 or DACS to compensate for their associated emissions is significantly cheaper than a system 18 dominated by intermittent renewables and energy storage. By offsetting $\mathrm{CO}_{2}$ emissions from 19 cheaper thermal plants, thereby allowing for their continued utilisation in a carbon-constrained 20 electricity system, BECCS and DACS can reduce the cost of decarbonisation by $37-48 \%$. Allowing 21 some this value transferred to accrue to NETs offers a potential route for their commercial 22 deployment.

Keywords: negative emissions technologies, BECCS, direct air capture

\section{Introduction}

Several studies have shown that the relationship between cumulative greenhouse gas (GHG) emissions and 26 global warming is insensitive to the emission pathway (i.e. the time and rate at which emissions occur) $\frac{112}{2} \quad 27$ Therefore, climate change risks and impacts can be mitigated by minimising historic emissions ${ }^{3}$. This 28
\end{abstract}

${ }^{*}$ Corresponding author: Tel: +44 (0)207 594 9298; E-mail: niall@imperial.ac.uk 
has generated interest in the potential for large-scale greenhouse gas (GHG) removal from the atmosphere 29 via negative emissions technologies (NETs). NETs, sometimes called carbon dioxide removal (CDR) 30 technologies, are technologies that can remove $\mathrm{CO}_{2}$, directly or indirectly, from the atmosphere ${ }^{415}$. A 31 portfolio of technologies has been identified as viable options to deliver negative emissions at scale. 32 These include: bio-energy with carbon capture and storage (BECCS) ${ }^{5}$, direct air capture and storage ${ }_{33}$ $(\mathrm{DACS})^{677}$, afforestation/reforestation $(\mathrm{AR})^{8}$, ocean fertilisation ${ }^{9.10}$, enhanced weathering of minerals ${ }^{[1-13}, \quad 34$ and biochar 814 .

Through the Paris Agreement, most nations committed to keeping average global temperature rise to well 36 below $2{ }^{\circ} \mathrm{C}$ (above pre-industrial levels) by $2100 \frac{15}{15}$. Global decarbonisation pathways consistent with this 37 target are reliant on the extensive deployment of NETs, usually BECCS ${ }^{5}$. The UNEP Emissions Gap Report 38 2017 states that NETs are necessary in addition to other mitigation options to manage possible overshoot of 39 near-term GHG emissions targets. In the UK, the Climate Change Act $2008^{16}$ (CCA) set a 2050 target to 40 reduce economy-wide greenhouse gas (GHG) emissions by $80 \%$ relative to 1990 levels. Fig. 1 1 illustrates 41 the projected reductions in sectoral emissions required to achieve this. The power sector must be essentially 42 completely decarbonised by $2050^{17}$.

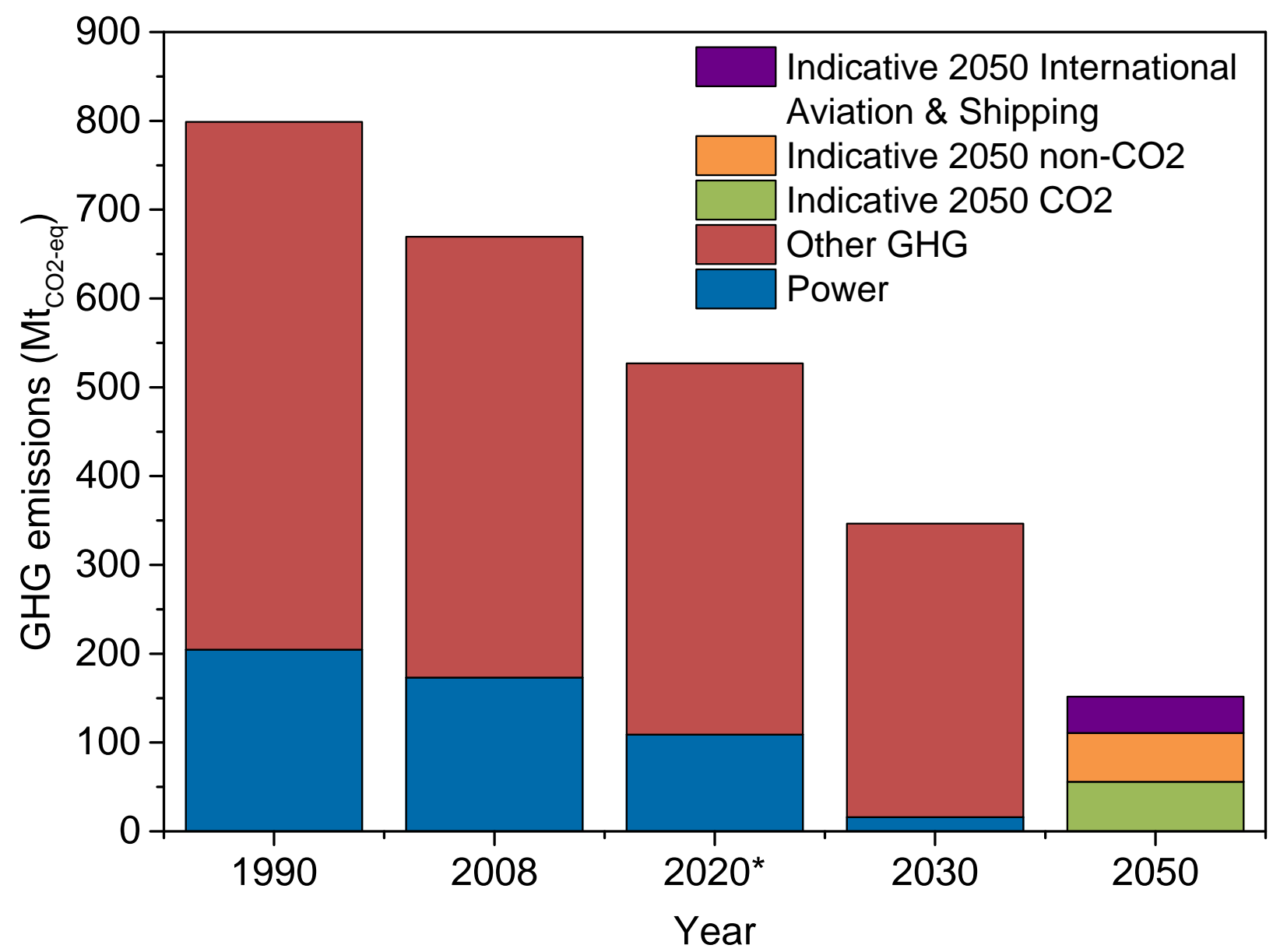

Figure 1: Power sector emissions in the context of UK greenhouse gas emissions (1990-2050). Figure adapted from CCC report.

*Assuming recommended implementation of measures proposed by the $\mathrm{CCC}^{17}$. 
To meet its commitments to the Paris Agreement, however, the UK requires deeper decarbonisation pathways (relative to the CCA target). Fig. 2 2 shows the sectoral emissions that must be achieved by 2050 to keep a $50 \%$ likelihood of keeping global temperature rise to $1.5^{\circ} \mathrm{C}$ by 2100 . Approximately $50 \mathrm{Mt}_{\mathrm{CO} 2} / \mathrm{y}$ of negative emissions are needed by 2050 to offset $\mathrm{CO}_{2}$ emissions from sectors with more difficult or expensive mitigation solutions, including aviation, shipping and transport 18 .
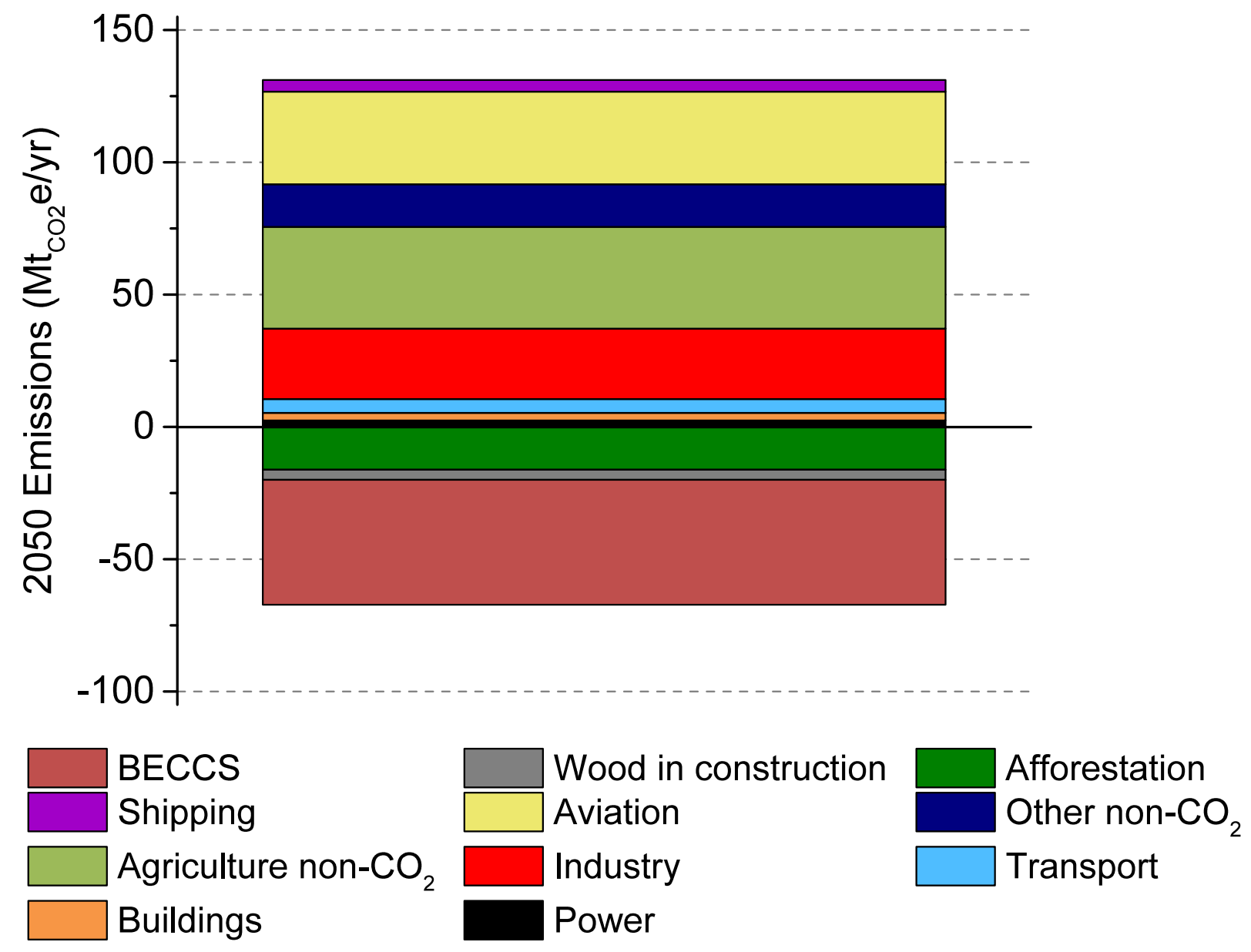

Figure 2: Residual UK greenhouse gas emissions in 2050 under the CCC's Max deployment scenario 18 . 92\% GHG emissions reduction (relative to 1990 levels) is achieved by 2050. This is within the range of $86-96 \%$ suggested by the most ambitious paths available from global climate-economy models (which keep a $50 \%$ likelihood of $1.5^{\circ} \mathrm{C}$ in 2100 after overshooting). Figure has been adapted from the CCC report 18 .

Whilst large-scale afforestation/reforestation has long been practised $\frac{19[20}{B^{2}}, \mathrm{BCCS}^{21}$ and DACS $22 \sqrt[24]{ }$ are the only technological NETs being pursued at demonstration scale. These technologies are the focus of this study. Studies that have evaluated the negative emissions achievable through deployment of BECCS or DACS have considered them in isolation ${ }^{25}$ and have neglected to consider the operation of these technologies within the broader energy system, and thus have not provided any insight into the extent to which they provide value to these systems, or the way in which they might operate therein. There is therefore a lack of understanding of the services they can provide and the incentives needed for each service (e.g., power generation, $\mathrm{CO}_{2}$ removal). 
This study investigates the potential role for BECCS and DACS in achieving the: 1) 2050 UK power sector decarbonisation target decreed by the Climate Change Act 2008 $\frac{16}{}$, and 2) negative emissions needed to be consistent with the Paris Agreement target, which is not legislated. It also quantifies the value added by BECCS and DACS to the UK energy system, and describes their operation within it.

Section 1.1 reviews the ongoing transition of the UK electricity system. Section 2 describes the Electricity Systems Optimisation (ESO) modelling framework used in this study and the parametrisation of NETs within the model. In section 3 , possible decarbonisation pathways for the power sector, subject to different emission targets and the availability of NETs, are outlined. The value of negative emissions is discussed in section 4. Lastly, sensitivity analysis of the results presented to key modelling parameters is given in section 5 and we present some conclusions in section 6 .

\subsection{The UK electricity system}

Fossil fuels have historically dominated the fuel mix for power generation in the UK and still provide the majority of power generation today. Fig. 3 shows that coal and gas contributed 53\% of the fuel input for electricity generation in 2016. Imminent emissions reductions targets have spurred a transition to less-polluting fuels and renewable sources of energy $16 / 26 / 27$. Most notably, a planned phase-out of coal-fired plants by $2025^{28}$ and an increase in the carbon price floor in 2015 (from $£ 9 / \mathrm{t}_{\mathrm{CO}_{2}}$ to $£ 18 / \mathrm{t}_{\mathrm{CO}_{2}}{ }^{29}$ ) has led to a rapid decline in coal's share of generation. In 2016, coal supplied $9 \%$ of electricity, down from $22 \%$ in $2015^{29}$. This decline has been compensated by increased gas-fired and renewable electricity generation, the latter enabled by policies and incentives favouring renewable energy sources 2730,33 .

Total electricity supply to the UK was 357 TWh in 2016, including net imports via interconnectors 29 . The UK has $4.1 \mathrm{GW}$ of interconnector capacity allowing trade with France $(2 \mathrm{GW})$, the Netherlands $(1 \mathrm{GW})$ and Ireland $(1.1 \mathrm{GW})^{34}$. By source, electricity supply was: $40 \%$ gas, $20 \%$ nuclear, $15 \%$ non-thermal renewables (natural flow hydro, wind, solar, wave and tidal), 9\% coal, $8 \%$ bio-energy (mostly from biomass co-fired with coal in Drax power station), $5 \%$ imports and $1 \%$ pumped hydro storage 29 . The rest is used by generators for their own plant works. Final electricity consumption was 304 TWh due to network losses (26 TWh) and consumption from within the energy industry $(27 \mathrm{TWh}) \underline{29}$.

Although unabated gas plants-gas plants not equipped with carbon capture and storage (CCS) technology-emit less than half of the $\mathrm{CO}_{2}$ of coal-fired plants, they still emit approximately 360-390 $\mathrm{kg}_{\mathrm{CO}_{2}} / \mathrm{MWh}$ of electricity generated ${ }^{29}$. As the UK seeks to transition to a decarbonised electricity system, unabated gas plants cannot continue to dominate the generation mix without being equipped with CCS and negative emissions to compensate for residual emissions from the CCS plants.

The variability in the supply of wind and solar energy poses challenges to the electricity system ${ }^{36}$. Limited transmission capacity and energy storage, and the relative inflexibility of base load generators (e.g., nuclear plants) result in curtailment of power generation from intermittent renewable energy sources (IRES) during periods of surplus generation and low demand $37 / 38$. This is a necessary security measure required to maintain power system stability and operability, and to prevent equipment damage ${ }^{39}$. In the UK, constraint payments at an electricity price of $£ 157 / \mathrm{MWh}$ are made to wind farms to curtail their generation $\frac{40}{\text {. In the }}$ absence of increased energy storage and flexibility in the electricity system, further penetration of IRES will see increased curtailment levels and hence, constraint payments. Approximately $2.7 \mathrm{GW}$ of pumped hydroelectric energy storage (PHES) provide all the bulk electricity storage in the UK currently 29 . Although there are plans to increase this by up to $2 \mathrm{GW}^{41}$, long-term expansion of PHES is limited by geography. Current plans are to install 7.3 GW of additional interconnection capacity by $2022^{34}$. This will add further flexibility to the electricity system, which will serve to minimise curtailment. 

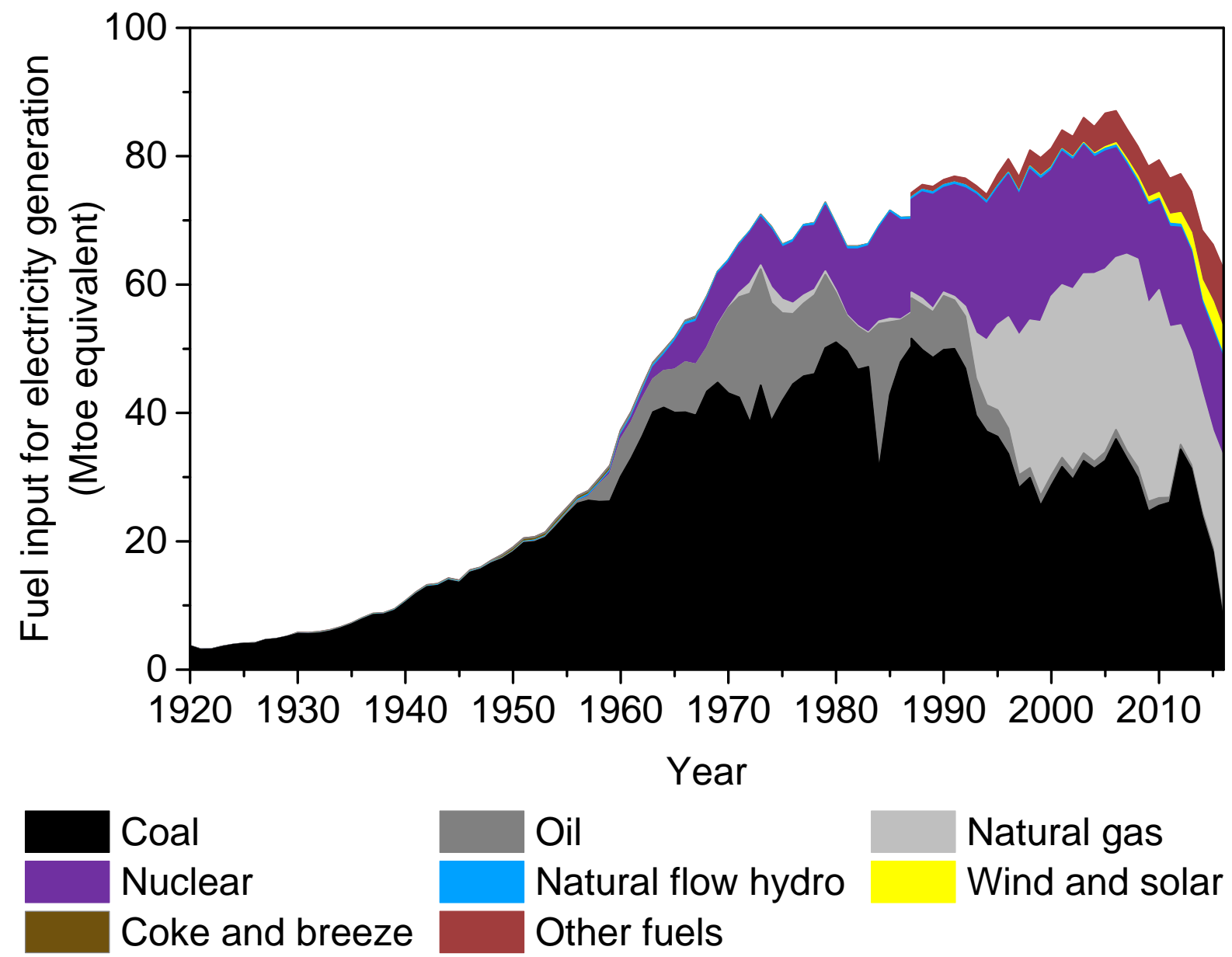

Natural gas

Wind and solar

Figure 3: Fuel inputs for electricity generation in the UK from 1920 to 2016 35. "Other fuels" include coke oven gas, blast furnace gas, waste products from chemical processes, refuse-derived fuels and other renewable sources including wind. Industrial hydro data are not available before 1951. Natural gas includes colliery methane from 1987 onwards.

The future role of nuclear power in the UK is threatened by its high upfront investment costs and lengthy construction times - the recently approved Hinkley Point C (HPC) plant will cost $£ 30$ billion and take 10 years to build ${ }^{42143}$. Its long payback periods leave it susceptible to government policy changes that may reduce revenues hence financing is difficult to raise ${ }^{42}$. HPC has since been shown to provide marginal value-for-money for consumers, mainly due to large financing costs ${ }^{44}$. Increasing costs of the technology due to more stringent safety requirements further compound to the difficulties $\underline{45 / 46}$.

The above highlights some of the challenges that the UK faces as it seeks to transition to a low-carbon electricity system. This study will focus on the integration of NETs into the future electricity system, their impact(s) on the optimal system design, including how the challenges discussed can be avoided or overcome.

\section{NETs in the electricity system}

Within the electricity system, NETs can generate net negative $\mathrm{CO}_{2}$ emissions to: 1) offset emissions from 110 unabated and abated fossil generators, and/or 2) offset emissions from sectors that have more difficult or 111 
costly mitigation solutions. To meet the commitments to the Paris Accord, it is estimated that $-47 \mathrm{Mt}_{\mathrm{CO}_{2}} / \mathrm{year} 112$ from BECCS is needed by 2050 in addition to afforestation, to offset non-power sector emissions (shown in 113 Fig. 2). Direct air capture of $\mathrm{CO}_{2}$ and subsequent storage (DACS) has since emerged as another potential 114 source of large-scale negative emissions. Both technologies are described in detail below. 115

\section{Bioenergy with carbon capture and storage (BECCS)}

Plants extract $\mathrm{CO}_{2}$ from the atmosphere during their growth and convert it into carbohydrates via photosynthesis. When burned, the $\mathrm{CO}_{2}$ captured is returned to the atmosphere hence net emissions from growth and combustion are zero. Biomass use for large-scale power or fuel production, however, requires processing due to its high moisture content and heterogeneous nature. Raw material and processed feedstock must also be transported to processing facility and the power plant, respectively. Land-use change (LUC), and in particular indirect LUC, may also be a significant contributor to GHG emissions, and these will likely need to be quantified on a case by case basis as they are dependent on a range of factors (location, type of land, etc. (47.49 $^{4}$. These processes comprise the biomass supply chain and each contributes an additional energy input and carbon footprint that must be considered in the life-cycle emissions of biomass. Integrating a dedicated biomass-firing plant with CCS allows for $\mathrm{CO}_{2}$ that would be otherwise released, to be captured and permanently sequestered, thereby can result in a net removal of $\mathrm{CO}_{2}$ from the atmosphere (subject to sufficiently low supply chain emissions), i.e. negative emissions. As CCS capture efficiency is often around $90 \%$, there are residual emissions from BECCS but the overall carbon balance is negative. Fig. 4 provides a simplified representation of the biomass supply chain from harvest to combustion in a power plant.

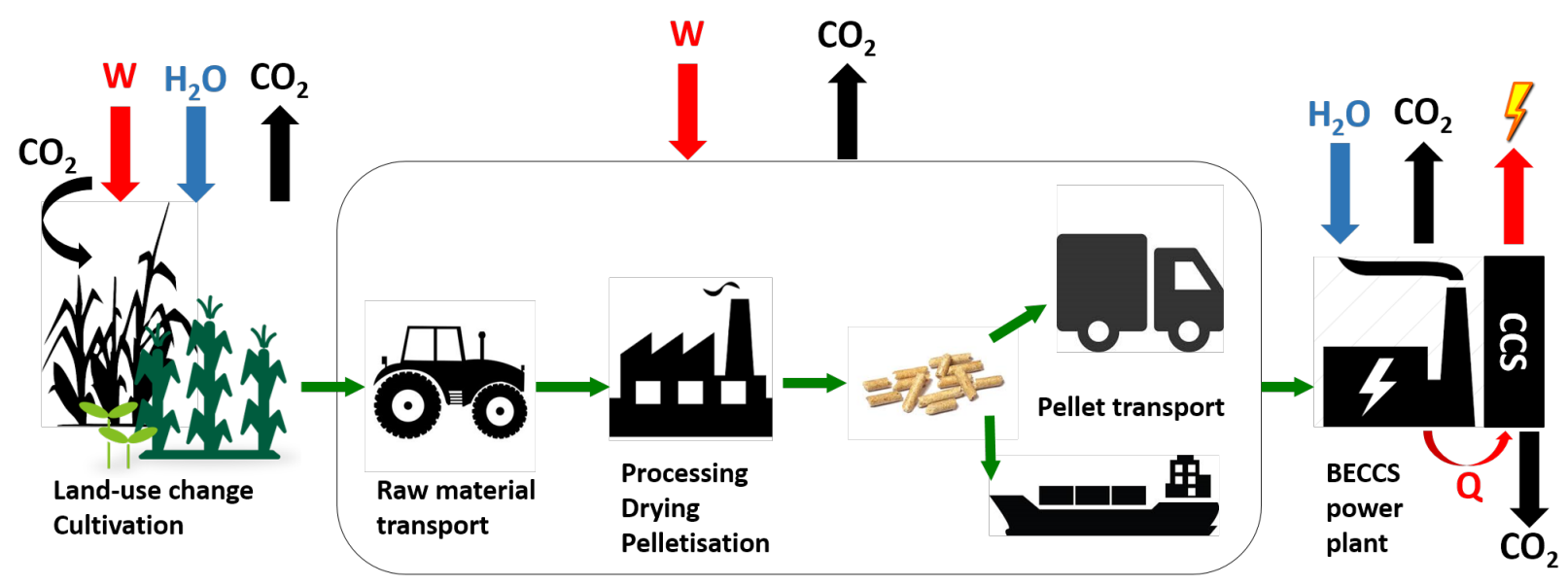

Figure 4: A simplified biomass supply chain from harvest to combustion in a BECCS power plant, showing inputs and outputs of $\mathrm{CO}_{2}$, water $\left(\mathrm{H}_{2} \mathrm{O}\right)$ and energy $(\mathrm{W}, \mathrm{Q})$ at different stages. This figure is adapted from 47.50 .

A study on the resource efficiency of BECCS has shown that its potential for negative emissions is largely-dependent on the biomass supply chain ${ }^{47}$. In this study, Miscanthus with associated supply chain GHG emissions of $39.2 \mathrm{~g}_{\mathrm{CO}_{2}-\mathrm{eq}} / \mathrm{MJ}_{\mathrm{e}}$ is assumed to be the feedstock to the BECCS plants; this is the mean value for a range of supply chains of UK-sourced Miscanthus 47 . These supply chain emissions are well below the sustainability limit in the UK $\left(79 \mathrm{~g}_{\mathrm{CO}_{2}-\mathrm{eq}} / \mathrm{MJ}_{\mathrm{e}}\right)^{51}$. We assume an ultra-supercritical power plant with post-combustion $\mathrm{CO}_{2}$ capture using conventional MEA and storage. Other technical features and costs of the BECCS plant are given in section 2.1. 
$\mathrm{CO}_{2}$ can be removed directly from the atmosphere by contacting the air with a range of sorbents. The ${ }_{139}$ low atmospheric concentration of $\mathrm{CO}_{2}$ (approximately 408 ppm ${ }^{52}$ ) means very large energy input and 140 volumes of air are required to obtain a pure $\mathrm{CO}_{2}$ stream. Commercial DACS technology manufacturers 141 typically employ amine-functionalised sorbents $\frac{23[53[54}{2}$ or wet-scrubbing systems with sodium or calcium 142 cycling $7722[55$. The archetype of DACS considered in this study uses a wet-scrubbing system with calcium 143 cycling, because of its potential ease of scale-up. It is illustrated in Fig. 5. A potassium or sodium hydroxide 144 solution captures $\mathrm{CO}_{2}$ in air and stores it as a carbonate. Calcium carbonate $\left(\mathrm{CaCO}_{3}\right)$ is then calcined in ${ }_{145}$ a kiln to produce calcium oxide $(\mathrm{CaO})$ and $\mathrm{CO}_{2}$. Hydrated $\mathrm{CaO}$ is then used to regenerate the hydroxide ${ }_{146}$ solution for further $\mathrm{CO}_{2}$ capture. The process description has been detailed previously ${ }^{22[55}$ and will not be ${ }_{147}$ repeated here. The majority of the energy input into DACS $-6.1 \mathrm{GJ}_{\mathrm{th}} / \mathrm{t}_{\mathrm{CO}_{2}}$ captured - is heat required for the ${ }_{148}$ calcination of $\mathrm{CaCO}_{3}$ at $900^{\circ} \mathrm{C}$. This is supplied via oxy-fired natural gas combustion, to avoid nitrogen 149 (from burning in air) in the capture exhaust which would require a further separation step. Approximately 150 $1.8 \mathrm{GJ}_{\mathrm{e}} / \mathrm{t}_{\mathrm{CO}_{2}}$ captured is required for fans, liquid pumping, air separation (to provide oxygen for combustion) 151 and $\mathrm{CO}_{2}$ compression $\frac{55}{5}$. DACS technology description within the modelling framework used in this study 152 is provided in section 2.1 .

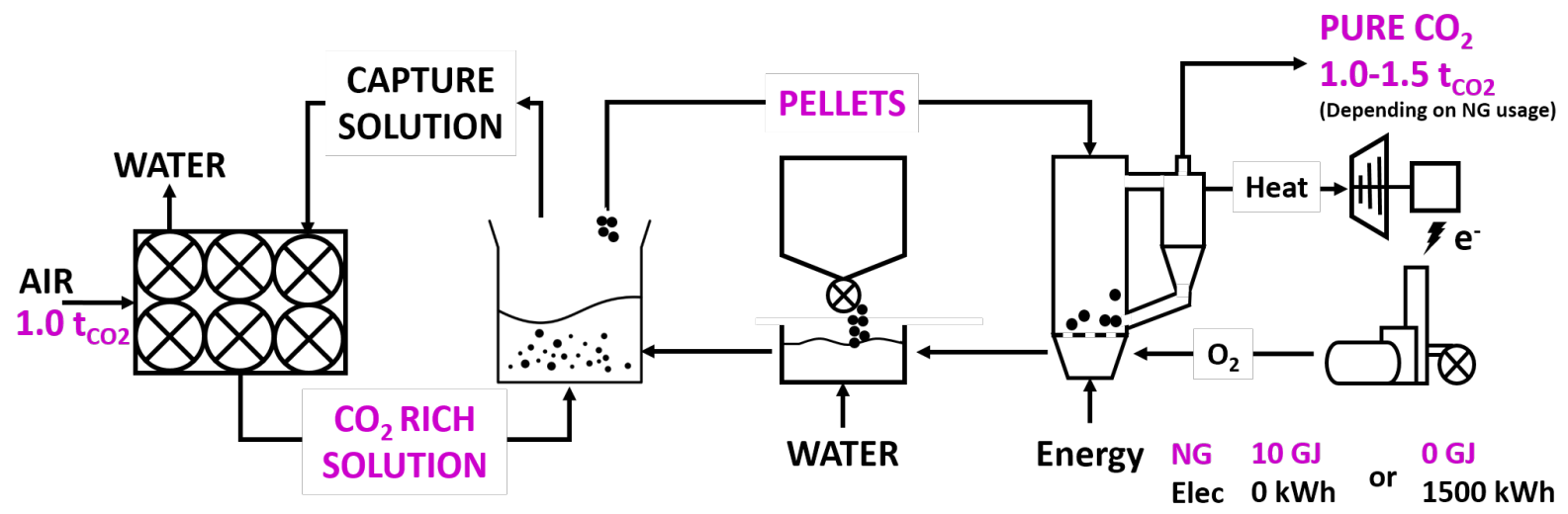

Figure 5: Simplified representation of a wet-scrubbing system for direct removal of $\mathrm{CO}_{2}$ from the air, adapted from ${ }^{22}$. Capture solution is often sodium hydroxide $(\mathrm{NaOH})$ or potassium hydroxide $(\mathrm{KOH})$. Pellets of calcium carbonate are used to regenerate the capture solution.

\subsection{Modelling}

The Electricity Systems Optimisation (ESO) modelling framework used in this study combines capacity 155 expansion planning and unit commitment model formulations to determine the optimal system design and 156 hourly dispatch of electricity in the UK. This work assumed an exogenously imposed electricity demand, 157 with an initial consumption of $304 \mathrm{TWh} / \mathrm{yr} \frac{56}{}$. A $1 \%$ year-on-year increase in electricity demand and an 158 unchanged demand profile, illustrated in Fig. 16, are assumed throughout the planning horizon considered. $\quad 159$

The ESO model assumes perfect foresight, and minimises the total system cost in the power supply system, 160 i.e. the capital and operational expenditure, subject to the constraints below:

- Capacity expansion constraints: initial capacity available is specified; technology-specific lifetimes, 162 maximum deployment (due to geographic or resource limits) and maximum build rates are specified. 
- System-wide constraints: electricity demand is always met; 4\% of peak load and 15\% of intermittent 164 power output must be available to provide reserve capacity; a minimum level of system inertia is 165 maintained to provide frequency and voltage control services*; maximum annual carbon emissions from 166 power generation are specified (discussed in section 1 .

- Technology constraints: power, reserve and inertia provision capability; flexibility of generation/storage units; carbon emissions; and technology-specific ramping (up and down) times are all specified. Appendix 2 details the technology costs and operational parameters implemented in the model.

The ESO model is a mixed-integer linear optimisation problem (MILP). The mathematical formulation of the model has been described previously 57,59 and is accessible as an open-source and open data model ${ }^{60}$. The original ESO formulation does not include DACS technology, and has been adapted to do so. Appendix 1 details the changes made.

The MILP formulation does not allow for the direct calculation of shadow prices, as this is dependent on the algorithmic approach used to solve the model ${ }^{61+63}$. The shadow price associated with a particular constraint represents the increase in the optimal value of the objective (in this case, total system cost) per unit increase in the amount of resources available. In this study, the marginal cost of electricity (MC) has been estimated using the shadow price of the electricity balance constraint (supply $=$ demand). Sensitivity analysis on the electricity demand (+/- $1 \mathrm{MW}$ ) was carried out to validate the MC estimates. The results of this are discussed in section 4

The planning horizon considered is 2015 to 2050, when the decarbonisation of the electricity system is to be achieved. A discount rate of $3 \%$ is used for future cash flows. The UK is assumed to be a single node, hence transmission and distribution constraints have not being taken into account explicitly, but overall transmission losses are included (as $7.7 \%$ of demand).

The power generating technologies considered were: Nuclear, Coal, Combined Cycle Gas Turbines (CCGT), Open Cycle Gas Turbine (OCGT), CCGT with post-combustion CCS (CCGT-CCS), BECCS, Onshore Wind, Offshore Wind, Solar PV, and Interconnector 7 Pumped Hydroelectric storage and generic grid-level storage, parametrised as Lead-Acid Battery, were considered as energy storage technologies. Due to the large energy input needed for DACS, it is modelled as a net consumer of power, i.e. it contributes additional electricity demand to provide negative emissions. As BECCS and DACS have only been proven at demonstration scale, they are made available for deployment at scale from 2030 onwards in the model. All other technologies are available from 2015. Appendix 2 details the technology costs and operational parameters assumed.

\subsubsection{BECCS}

The reference BECCS technology assumed uses Miscanthus pellets fired in a $500 \mathrm{MW}$ ultra-supercritical power plant with post-combustion capture using conventional MEA solvent $\left(\eta_{\text {net }}=35 \%\right)^{64}$. This plant provides approximately $0.80 \mathrm{t}_{\mathrm{CO}_{2}} / \mathrm{MWh}$ of negative emissions ${ }^{47}$. The load factor range for stable generation is $30-85 \%$, as for a conventional coal-fired plant. BECCS is yet to be developed at utility-scale, so costs cited in the literature are estimates. Eq. 1 was used to estimate the capital costs (CAPEX) of the BECCS technology considered. The CAPEX of an ultra-supercritical coal-fired plant with post-combustion CCS ( $£ 2368 / \mathrm{kW}^{65}$ ) was added to the cost of converting coal-fired generating units to full biomass-firing.

\footnotetext{
* system inertia is the amount of kinetic energy stored in the spinning parts of synchronous generators

†The UK's current interconnectors with Europe were modelled as infinitely-flexible power generators, with limits on generation capacity.
} 
This comprises modifications to fuel mills and boilers, and construction of pellet storage facilities; it is 203 approximately $£ 354 / \mathrm{kW}^{66 \text { t }}$

$$
C A P E X_{\mathrm{BECCS}}=C A P E X_{\mathrm{Coal}-\mathrm{CCS}}+C A P E X_{\text {Biomass-conversion }}
$$

There are approximately 8.4 Mha of grassland available in the UK 67 . Excluding 6.1 Mha necessary for 205 livestock production $\frac{68}{6}$, there is 2.3 Mha of land potentially available for Miscanthus production. With 206 average yield of 10 tonnes per hectare per year ${ }^{69}, 21 \mathrm{Mt}$ of pellets can be produced locally (after taking into 207 account the losses that occur during processing and transport). Locally-sourced Miscanthus raw material 208 costs $£ 49 /$ tonne ${ }^{70}$, however transport and processing increases the pellet cost to $£ 118 /$ tonne. This comprises 209 majority of the operational costs (OPEX) for the BECCS plant. Appendix 2 details the additional costs and 210 carbon emissions incurred along the biomass supply chain. Fig. 6 shows the contribution of the different 211 supply chain processes to the final pellet price. Imported pellets are assumed to cost $£ 190 /$ tonne ${ }^{71}$. This 212 higher cost has also been taken into account in the model when BECCS biomass demand exceeds domestic 213 supply. $\mathrm{CO}_{2}$ transport and storage costs of $£ 10 / \mathrm{t}_{\mathrm{CO} 2}{ }^{72]}$ have been assumed, although power plant and storage 214 siting are not explicitly considered in the model. Other parameters considered are provided in Table $1 . \quad 215$

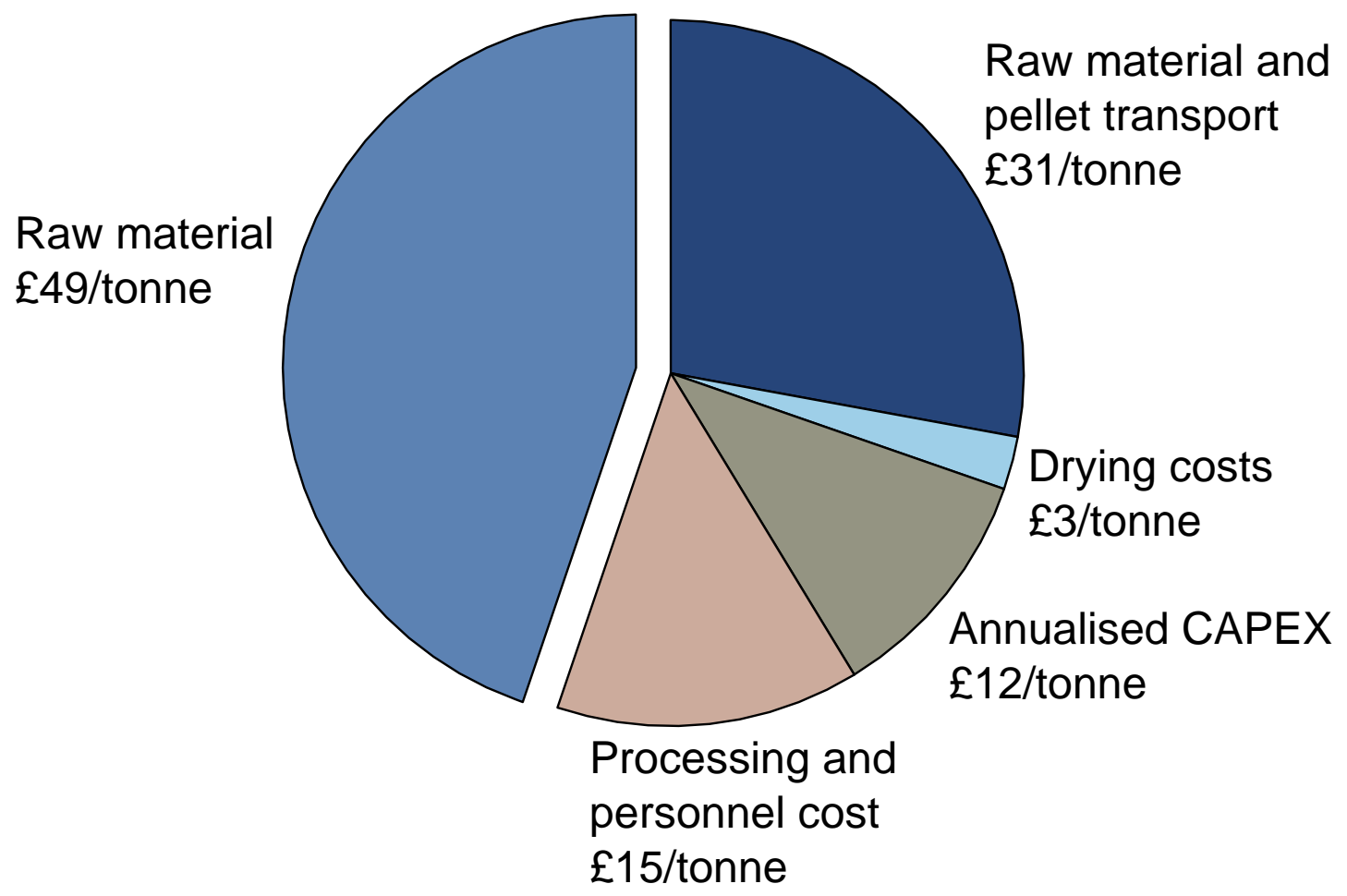

Conversion rate of $83.7 \%$ brings Miscanthus pellet price to $£ 118$ per tonne

Figure 6: Cost composition of UK grown and processed Miscanthus pellets.

\footnotetext{
${ }^{\ddagger}$ Based on $£ 700$ million conversion cost of three 660 MW units to full biomass-firing by Drax Power
} 


\subsubsection{DACS}

The DACS process considered in this work is a hydroxide-based capture using calcium cycling to regenerate the capture solution. The regeneration requires the calcination of calcium carbonate $\left(\mathrm{CaCO}_{3}\right)$, which occurs at approximately $900^{\circ} \mathrm{C}$. This does not allow for flexible operation as repeated cooling and heating of the kiln may threaten process stability. Therefore, it has been assumed that the plant is operated continuously at maximum $\mathrm{CO}_{2}$ removal rate (utilisation factor of $100 \%$ ). Based on the figures presented in section 2 . a DACS plant removing $1 \mathrm{Mt}_{\mathrm{CO}_{2}} / \mathrm{y}$ from the atmosphere requires $56 \mathrm{MW}_{\mathrm{e}}$ input. This is equivalent to a $\mathrm{CO}_{2}$ removal rate of $2 \mathrm{t}_{\mathrm{CO}_{2}} / \mathrm{MWh}$ consumed 55 . The bulk of the energy input to the air capture process, however, is high-grade heat to the kiln which is assumed to be supplied via oxy-fired natural gas combustion. Therefore the heat input does not contribute additional electricity demand to the system. DACS systems might potentially be composed of a number of small and modular $\mathrm{CO}_{2}$ collectors $23 \mid 24$. Consequently, the cost of $\mathrm{CO}_{2}$ collection, transport and storage is expected to be higher than for conventional power plants with CCS. This has been included as an added OPEX of $£ 30 / \mathrm{tO}_{2}{ }^{174}$. Other DACS technology costs were taken from the APS report ${ }^{55}$.

Table 1: Description of BECCS and DACS in the ESO modelling framework. Note that DACS is a net consumer of power, therefore its unit capacity is shown as negative. DACS CAPEX ${ }^{[55}$ has been levelised against the electricity consumption of the plant only; heat consumption not included.

\begin{tabular}{|c|c|c|}
\hline & BECCS & DACS \\
\hline Unit capacity (MW) & 500 & -56 \\
\hline Efficiency (HHV basis) & $35 \%$ & $7 \%$ \\
\hline Economic lifetime (years) & 30 & 25 \\
\hline Plant load factor & $30-85 \%$ & $100 \%$ \\
\hline Inertia potential (MW.s/MW) & 10 & - \\
\hline $\mathrm{CO}_{2}$ emissions rate $\left(\mathrm{t}_{\mathrm{CO}_{2}} / \mathrm{MWh}\right)$ & -0.8037 & -2.022 \\
\hline CAPEX (£/kW) & 2,721 & 33,074 \\
\hline Fixed OPEX (£/hour) & 4229 & - \\
\hline Variable OPEX (£/MWh) & $96-115$ & 260 \\
\hline Start-up OPEX (£/hour) & $4,000,000$ & - \\
\hline $\mathrm{CO}_{2}$ transport \& storage $\left(£ / \mathrm{t}_{\mathrm{CO}_{2}}\right)$ & 10 & $30^{74}$ \\
\hline UK pellet costs (£/tonne) & 118 & - \\
\hline Imported pellet costs (£/tonne) & 190 & - \\
\hline Natural gas price (£/MMBTU) & - & 3.74 \\
\hline Electricity price (£/MWh) & - & 44 \\
\hline
\end{tabular}

The description of the other technologies, fuel prices and carbon prices considered in this study are provided 230 in Appendix 2. The ESO model was used to determine the least-cost optimal capacity expansion for the UK 231 electricity system for the following scenarios:

- power sector decarbonisation by 2050 with: 1) no negative emissions technologies available to the system, 233 i.e. "No NETs", 2) DACS technology, but not BECCS, available to the system, i.e. "DACS Only", 3) 234 BECCS technology, but not DACS, available to the system, i.e. "BECCS Only" 235

- power sector providing $50 \mathrm{Mt}_{\mathrm{CO}_{2}}$ of negative emissions to meet commitments to the Paris Agreement (as 236 in Fig. 2), i.e. "1.5 ${ }^{\circ} \mathrm{C}$ system". Both BECCS and DACS are made available as the target cannot be met ${ }_{237}$ without NETs. 


\section{What might the future look like?}

\subsection{Zero emissions by 2050}

\subsubsection{No NETs}

Figures 7 and 8 show the optimal capacity and electricity generation mix, respectively, to achieve power sector decarbonisation by 2050 for the scenarios discussed in section 2.1. We observe that complete decarbonisation of the electricity system - defined here as a carbon intensity of less than $10 \mathrm{~kg}_{\mathrm{CO} 2} / \mathrm{MWh}$ - is achievable by 2050 without NETs. Such a scenario would see installed capacity increase by $208 \%$, from $98 \mathrm{GW}$ in 2015 to $301 \mathrm{GW}$ in 2050, due to the extensive expansion of IRES and nuclear power. Solar dominates the capacity mix in 2050, making up $30 \%(90 \mathrm{GW})$, but satisfies only $11 \%$ of annual demand due to its low energy density. Offshore and onshore wind contribute $30 \%$ and $19 \%$ of demand, respectively. As the existing nuclear plants reach the end of their lifetimes in the next decade, $24 \mathrm{GW}$ of new nuclear is added between 2030 and 2050 and its share of generation rises to $30 \%$ by mid-century.

Power plant utilisation factor, $U F_{i}$, is defined as the power output from a power plant $i$ divided by the maximum possible power output during a given time period. Eq. 2 defines the annual utilisation factor for plant $i$.

$$
U F_{i}=\frac{\text { annual power out put }(M W h)}{\text { plant capacity }(M W) \times 24 \text { hours } \times 365 \text { days }}
$$

The absence of negative emissions results in the underutilisation of unabated gas plants by 2050, as there is no $\mathrm{CO}_{2}$ removal to compensate for their emissions. By 2050, $\mathrm{UF}_{\mathrm{CCGT}}$ and $\mathrm{UF}_{\mathrm{OCGT}}$ fall to $<0.5 \%$, despite having $29 \mathrm{GW}$ of capacity installed. Unabated gas plants, however, provide $30 \%$ of the reserve capacity requirements in the system. Abated gas plants (CCGT-CCS) are also aggressively deployed from 2025, with $15 \mathrm{GW}$ added over the next 15 years. UF CCGT-CCS decreases from $69 \%$ initially to $16 \%$ in 2050 , as residual emissions from the CCS are minimised to meet the decarbonisation objective.

The dominance of IRES in this scenario leads to greater requirement for grid flexibility leading to increased energy storage and interconnector capacity installed. Storage service to demand peaks at $11 \mathrm{TWh} / \mathrm{y}$ in 2035 - a twofold increase from current levels. $9 \mathrm{GW}$ of additional interconnector capacity are also built, with their share of electricity supply peaking at $18 \%$ in 2025 , and subsequently falling to $4 \%$ by 2050 . This is because electricity imports have an associated carbon footprint ${ }^{75}$ which is attributed to the UK electricity system. As emissions targets become more stringent towards 2050, interconnection utilisation falls to minimise these associated emissions. Despite the increase in energy storage and flexibility services, incidences of curtailment still abound - $99 \mathrm{TWh} / \mathrm{y}$ ( $22 \%$ of demand) of IRES are curtailed in 2050. This is a lower-bound estimate as the model does not account for transmission congestion constraints.

While the No NETs system described is technically feasible, it is dominated by capital-intensive technologies. This is reflected in the large total system cost (TSC) - $£ 307$ billion of investment (CAPEX and OPEX) is needed in the power sector over 35 years. Fig. 10 illustrates the range of marginal costs of electricity (MC) for each scenario considered. In all scenarios, MCs are seen to be consistently higher than contemporary levels till 2050, when they peak. Peak MC is highest when no NETs are available to the system - it is approximately $£ 200 / \mathrm{MWh}$ in 2050. This is because new IRES and energy storage capacity is required to provide an additional MWh of carbon-neutral electricity, as current capacity is maximally utilised. Underutilised (and cheaper) unabated or abated gas power plants cannot provide the electricity due to the decarbonisation constraint. The rapid expansion of IRES capacity must also be accompanied by expansion of transmission and distribution (T\&D) networks, which incurs additional costs that are not 


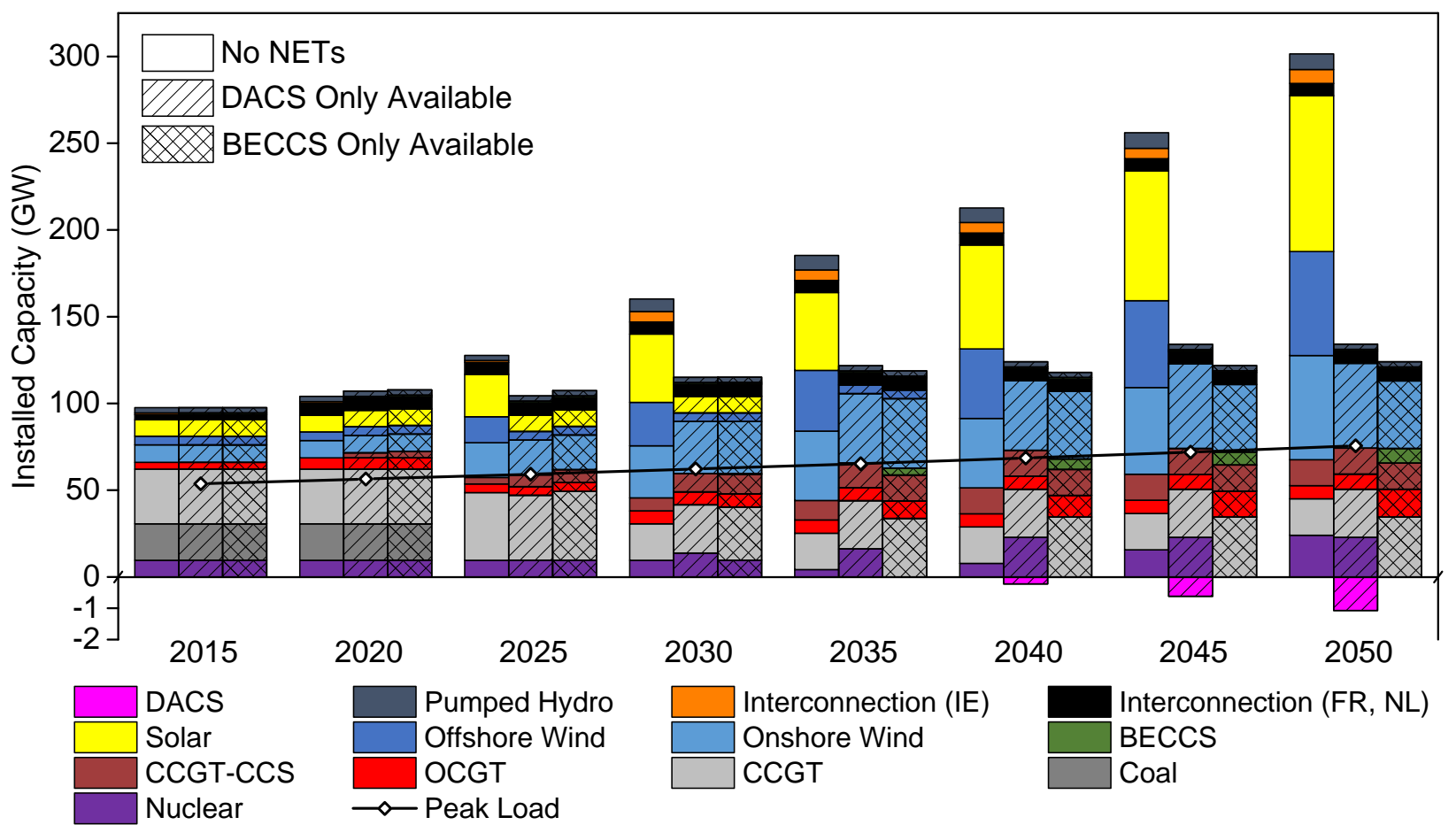

Figure 7: Optimal installed capacity mix for the UK, given the availability of negative emissions technologies for large-scale deployment.

included in the total system cost. Should constraint payments to IRES generators continue, curtailment will 279 also contribute to additional costs.

\subsubsection{DACS Available}

The availability of direct air capture and storage is observed to reduce the cost of complete decarbonisation 282 considerably. Despite the costliness of DACS, it is seen to be deployed from 2040 and to reduce the total investment needed to $£ 193$ billion (Fig. 9), 37\% less than with No NETs. This is because the negative emissions from DACS allow for continued utilisation of cheaper thermal plants. DACS consumes $9.4 \mathrm{TWh} / \mathrm{y}$ ( $2 \%$ of projected annual electricity demand) by 2050 to provide $19 \mathrm{Mt}_{\mathrm{CO}_{2}} / \mathrm{y}$ of negative emissions that offset $\mathrm{CO}_{2}$ emissions from unabated and abated gas plants (shown in Fig. 13). The offset allows for increased gas utilisation; $U F_{C C G T}$ doubles to $14 \%$ from 2040 to 2050 (period when DACS is operational), and $U F_{C C G T-C C S}$ increases to $>70 \%$ in the same period - a fourfold increase from its utilisation when no NETs are available.

Increased power generation from gas plants also completely displaces the need for new Solar and Offshore Wind, with no new capacity built after the existing plants reach their end life. However, $49 \mathrm{GW}$ of new Onshore Wind capacity is added by 2050. Consequently, IRES share of generation falls from 70\% (when no NETs are available) to $36 \%$, and energy storage requirements remain at contemporary levels. Reduced penetration of IRES also reduces flexibility needs with only $4 \mathrm{GW}$ of interconnector capacity added. The availability of negative emissions to compensate for 'imported emissions', however, results in increased interconnector utilisation and hence reliance on electricity imports. Imports satisfy 9-18\% of demand, up to three times its current share. The $24 \mathrm{GW}$ of nuclear build after 2030 remains necessary to meet the bulk of electricity demand $-159 \mathrm{TWh} / \mathrm{y}$ by 2050 (35\%). This share peaks at 39\% in 2040 before falling slightly. Therefore to achieve power sector decarbonisation, a system dominated by firm and dispatchable low-carbon 


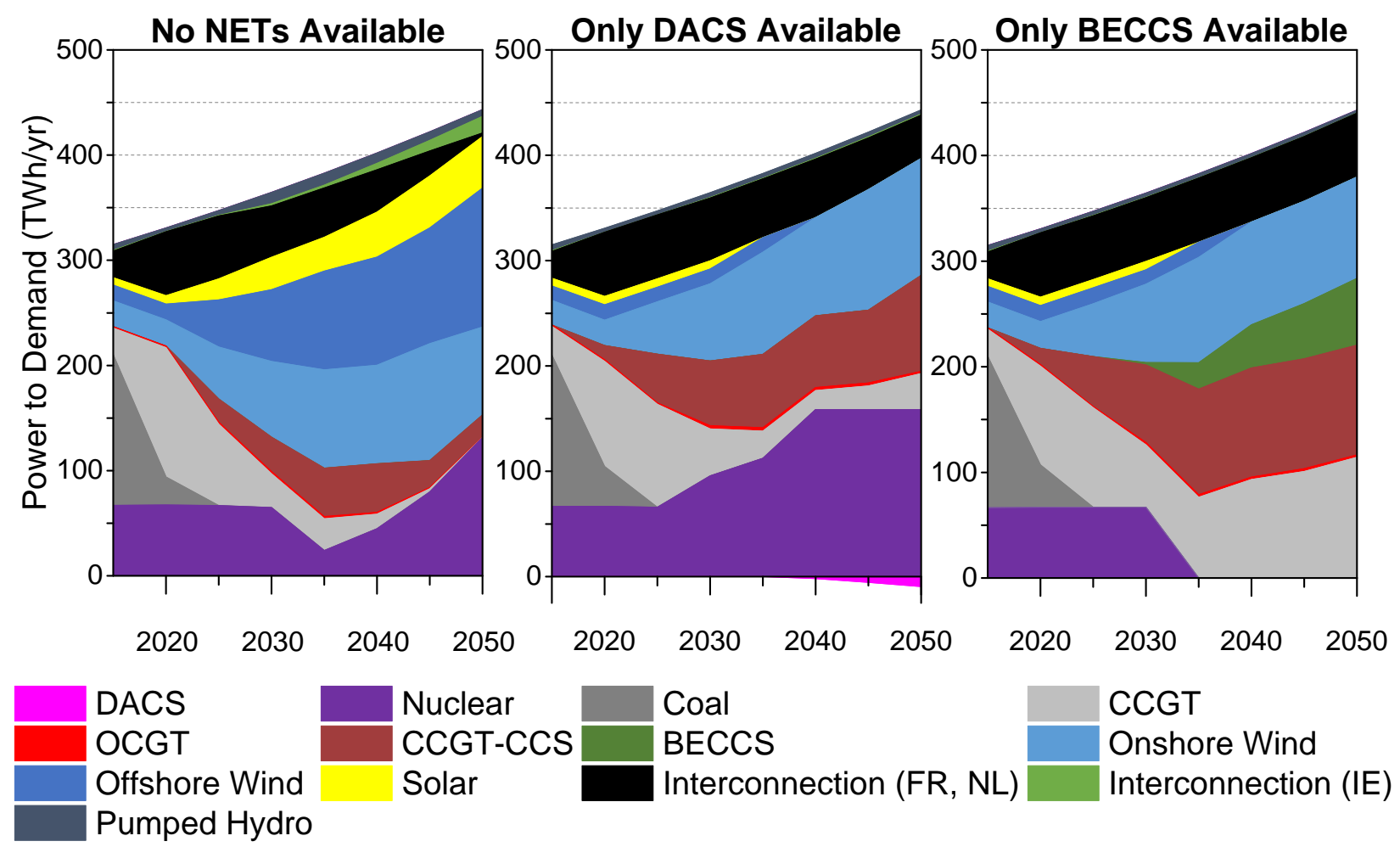

Figure 8: Optimal electricity generation mix for the UK from 2015 to 2050, when a zero emissions target is to be achieved, given the availability of negative emissions technologies for large-scale deployment.

generators with DACS to compensate for their associated emissions is significantly cheaper than a system 300 dominated by IRES and energy storage.

Annual MCs are seen to be similar with and without DACS availability. Similar peaking at £206/MWh 302 occurs in 2050, approximately four times the current average electricity cost of $£ 47 / \mathrm{MWh}^{76}$. The high MC ${ }_{303}$ in 2050 occurs due to the large OPEX of DACS plants. A marginal increase in demand is satisfied by the 304 increased operation of cheap thermal plants or imports (Nuclear and IRES are already maximally utilised) 305 and DACS is needed to compensate for the resulting emissions, which in turn results in an added electricity 306 demand.

The challenges surrounding social attitudes to land-based IRES ${ }^{77}$, particularly Onshore Wind, will remain 308 as installed capacity increases fivefold by 2050. Issues surrounding the feasibility of future conventional 309 nuclear projects and CCS, discussed in section 1.1, also persist. Lastly, an increased reliance on electricity 310 imports may also raise energy security concerns.

\subsubsection{BECCS Available}

When BECCS is made available to the system, total investment needed in the power sector by 2050 falls 313 to $£ 160$ billion, a $48 \%$ decrease relative to the No NETs scenario. Therefore, BECCS deployment provides 314 the greatest reduction in total system cost. We observe that $8.5 \mathrm{GW}$ of BECCS capacity are built by 2050315 to provide $51 \mathrm{Mt}_{\mathrm{CO}_{2}} / \mathrm{y}$ of negative emissions. This offsets emissions from gas plants and interconnectors, 316 thereby allowing $U F_{C C G T}$ and $U F_{C C G T-C C S}$ to rise to $38 \%$ and $79 \%$, respectively. In addition to the $\mathrm{CO}_{2} \quad 317$ 


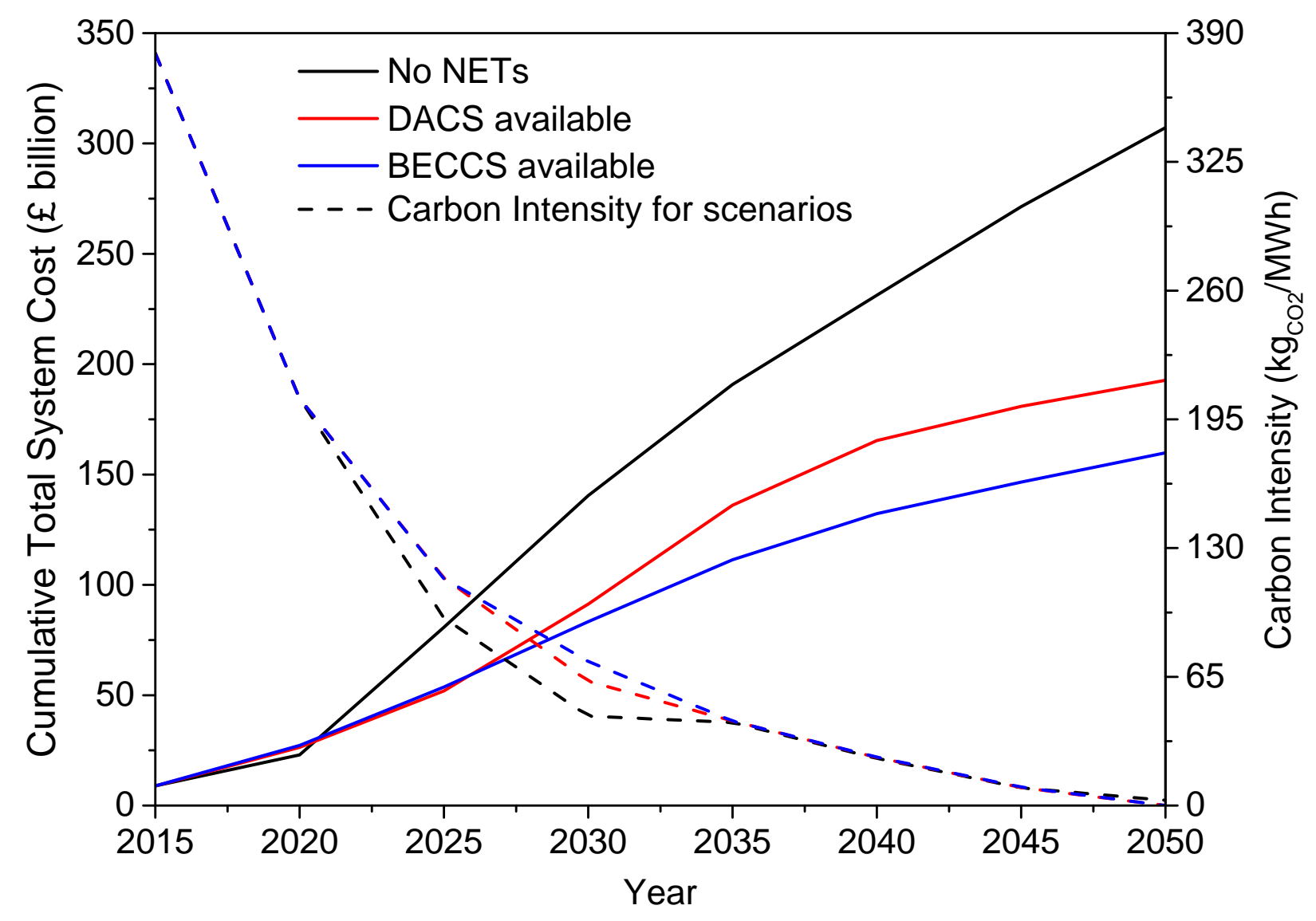

Figure 9: The cumulative total system cost of an optimal UK electricity system form 2015 to 2050, given the availability of negative emissions technologies for large-scale deployment.

removal service it provides, BECCS produces $63 \mathrm{TWh} / \mathrm{y}$ by 2050. Together, BECCS and gas satisfy $64 \%$ of 318 demand: CCGT (26\%), CCGT-CCS (23\%), BECCS (14\%) and OCGT (0.5\%). Increased generation from 319 thermal plants displaces more expensive low-carbon electricity generators from the system. No new Nuclear, 320 Solar or Offshore Wind power plants are built after the existing fleet reach the end of their operational 321 lifetimes. However, $39 \mathrm{GW}$ of Onshore Wind is added by 2050, resulting in an increase of IRES penetration 322 from $25 \%$ of capacity installed today to $31 \%$ in 2050 . No added energy storage is needed to accommodate 323 increased IRES penetration $-3 \mathrm{GW}$ of pumped hydroelectric storage currently available however continue 324 to provide $0.6 \%$ of demand.

The generation of $63 \mathrm{TWh} / \mathrm{y}$ of electricity by BECCS requires $12 \mathrm{Mt}_{\text {pellets }} / \mathrm{y}$ of fuel-grade biomass, assumed 326 to be provided by Miscanthus in this work. Domestic Miscanthus supply $-21 \mathrm{Mt}_{\text {pellets }} / \mathrm{y}$ - can satisfy the 327 biomass demand and more expensive pellet imports are not required. The necessity for a sustainable supply 328 of biomass in order for BECCS to result in net removal of $\mathrm{CO}_{2}$ from the atmosphere has been discussed at ${ }_{329}$ length elsewhere ${ }^{47.78}$, and as such are not discussed further here.

In this scenario, MC consistently rises until 2050 when it peaks at $£ 109 / \mathrm{MWh}$, more than twice the current ${ }_{331}$ price. This is however approximately half of the MC observed in the No NETS or DACS Only scenarios. 


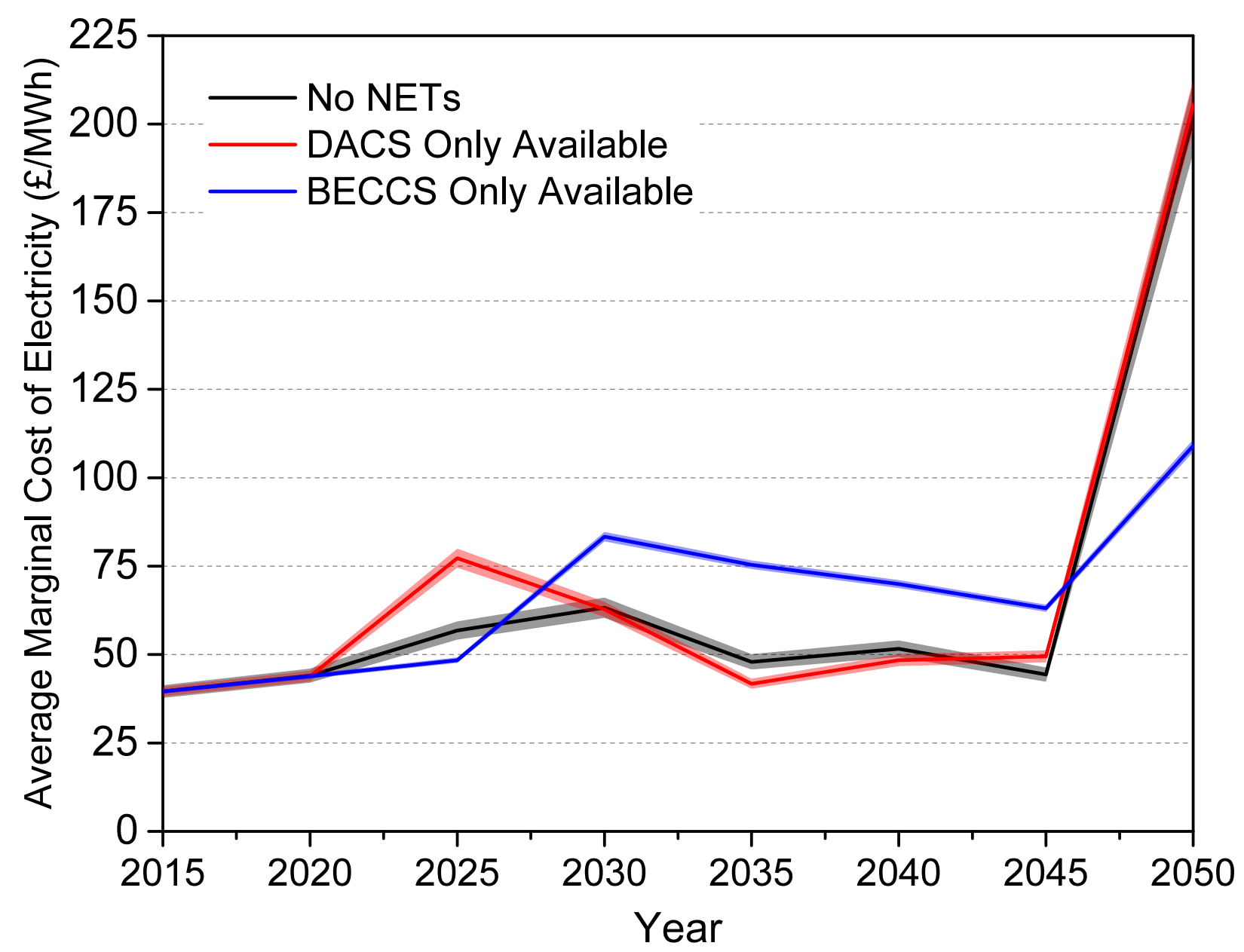

Figure 10: Average annual marginal cost of electricity in the UK depending on the deployment of BECCS or DACS in the system.

\subsection{A " $1.5{ }^{\circ}$ C system"}

The emissions target for 2050 is adjusted to $-50 \mathrm{Mt}_{\mathrm{CO}_{2}} / \mathrm{y}$ in the model, so the UK can meet its commitment to the Paris Agreement $\frac{18}{}$, illustrated in Fig. 2. BECCS and DACS are also made available for deployment from 2015. All other model parameters remain unchanged. Fig. 11 illustrates the electricity supply mix necessary to meet this target by 2050 . We observe that at least $93 \mathrm{Mt}_{\mathrm{CO}_{2}} / \mathrm{y}$ of negative emissions are needed by 2050 - $43 \mathrm{Mt}_{\mathrm{CO}_{2}} / \mathrm{y}$ compensates for the fossil generators in the system while the rest serves as available offset for other sectoral emissions. This is all provided by BECCS as it has cheaper abatement cost $\left(£ 190 / \mathrm{t}_{\mathrm{CO}_{2}}\right)$ compared to DACS ( $£ 390 / \mathrm{t}_{\mathrm{CO}_{2}}$ ), and can contribute to electricity supply.

To satisfy the $\mathrm{CO}_{2}$ removal target above, approximately $23 \mathrm{Mt} / \mathrm{y}$ of Miscanthus pellets are needed by 341 2050. This is greater than current projections of domestic biomass availability, thus necessitating the 342 import of potentially more expensive biomass. The optimal electricity generation mix needed to achieve 343 the Paris Agreement target is shown in Fig. 11. This system requires $£ 176$ billion to be invested in the 344 power sector from 2015 to 2050, a 10\% increase compared to the BECCS Only scenario. BECCS capacity 345 installed in 2050 increases from 8.5 to $15.5 \mathrm{GW}$ as more negative emissions are required to meet the deeper 346 decarbonisation target. The corresponding increase in generation displaces further unabated gas generation. 347 
Consequently, less negative emissions are needed to offset ossil-derived emissions, thereby allowing more 348 offset for non-power sectoral emissions. By 2050, unabated gas capacity is $46.8 \mathrm{GW}, 3.8 \mathrm{GW}$ less than 349 installed capacity when mid-century decarbonisation is the objective. CCGT utilisation, $U F_{C C G T}$ also falls 350 from $38 \%$ to $31 \%$.
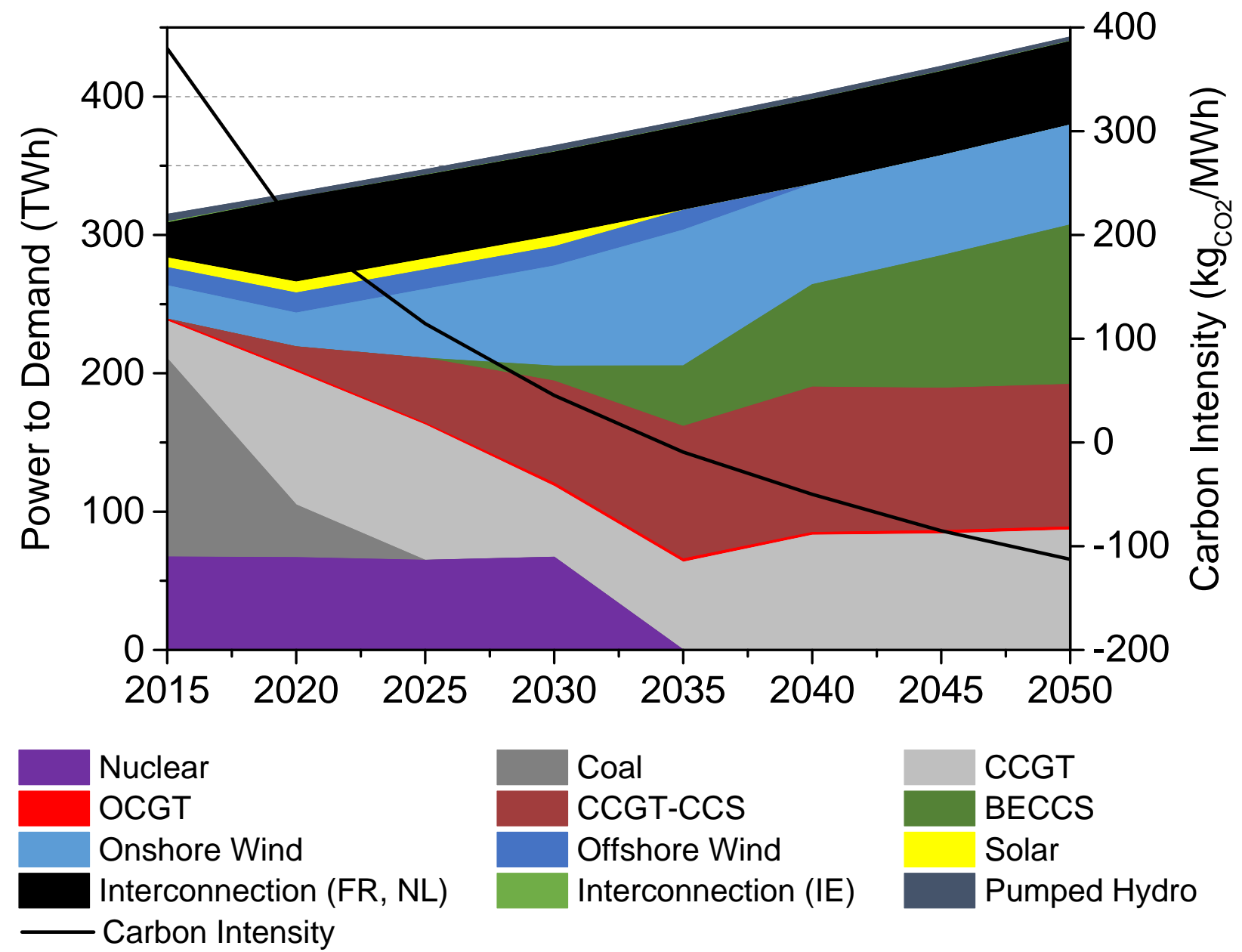

Figure 11: Optimal electricity generation mix for a UK electricity system consistent with the Paris Agreement target.

Nuclear, Solar and Offshore Wind are phased out as the existing power plants are not replaced at the end of their lifetime, while $30 \mathrm{GW}$ of new Onshore Wind capacity is added by 2035. $15 \mathrm{GW}$ of CCGT-CCS are deployed as in all scenarios previously discussed. Share of generation in 2050 is $26 \%$ BECCS, $23 \%$ CCGT-CCS, 20\% CCGT, 16\% Onshore Wind, 14\% Imports, 0.7\% Pumped Storage, and 0.5\% OCGT. Therefore, should BECCS technology be available for deployment, it would need to supply more than a quarter of the UK's electricity demand to provide sufficient negative emissions for compliance with the Paris Agreement. Furthermore, such a scenario would see biomass and electricity imports meet approximately $16 \% \$$ of demand, a threefold increase in import's share of generation today.

Onshore Wind features significantly in all the scenarios discussed, satisfying 16-36\% of demand. More 360 expensive Offshore Wind, however, is phased out in all but the No NETs scenario, as existing capacity is not

\footnotetext{
$\S 14 \%$ from interconnectors and $2 \%$ from imported biomass
} 
replaced after it reaches the end of its lifetime. Only lower cost IRES are therefore valuable to the system. 362 The most recent auction in the UK saw falling costs of Offshore Wind ${ }^{79}$. In addition, public attitudes may 363 favour unseen Offshore Wind farms over (new) onshore farms that disrupt landscapes. Reduced availability 364 of Onshore Wind and cheaper Offshore Wind may therefore lead to the deployment of Offshore Wind in the 365 future, contrary to what we observe here.

We also observe direct competition between BECCS and Nuclear, as BECCS deployment displaces the need for new nuclear plants in the system. Therefore, flexible carbon-negative generation to augment gas generation, and offset gas-derived emissions (from CCGT and CCGT-CCS), proves more valuable to the system than base load zero-carbon generation.

\section{What is the value of negative emissions?}

The reductions in system costs and marginal electricity costs achievable due to the availability of NETs have been shown to be significant, especially for BECCS. To quantify the value that each technology adds to the system, two metrics have been employed: the System Value ${ }^{57}$ and the Value Transfer, as introduced in this work.

\subsection{System value}

The System Value (SV) metric quantifies the value of a technology, $i$, to the power system as the reduction in total system cost (TSC) caused by the deployment of the technology 57 . This is shown in Eq. 3. The reference year from which the reduction in TSC is calculated is 2050, when the decarbonisation objective is to be met.

$$
S V_{i, k}=\frac{T S C_{i, k=0}-T S C_{i, k}}{k_{i}}
$$

where $T S C_{k}$ is the total system cost (TSC) in 2050 for a given deployment, $k(\mathrm{~kW})$, of technology $i$, and $T S C_{i, k=0}$ is the TSC in 2050 when technology $i$ is not available to the system. As DACS is a net consumer of heat and power, $k_{D A C S}$ is the installed capacity required to meet its energy needs. An electricity-to-heat conversion factor of $45 \%$ was used to determine the installed capacity required to satisfy its energy input. Hence its $\mathrm{SV}$ is given in $£ / \mathrm{kW}_{\text {required }}$.

The available capacity of BECCS and DACS for deployment was increased until there was no marginal decrease in TSC. That is, should more of the technology be made available, it is not deployed as the resulting capacity mix is not cost-optimal. This limit is called the economic level of deployment (EL) of the technology, and is discussed in detail in our previous work ${ }^{57}$. The System Value curves for both BECCS and DACS are illustrated in Fig. 12. On initial deployment, BECCS has an SV of approximately $£ 124,000 / \mathrm{kW}$, three times that of DACS - £43,000/kW $\mathrm{k}_{\text {required. }}$ As more BECCS is made available, the marginal reduction in TSC per installed capacity falls until BECCS reaches its EL. BECCS and DACS were found to have an ELs of $8.5 \mathrm{GW}_{\text {installed }}$ and $8.4 \mathrm{GW}_{\text {required }}$, respectively. As can be observed from Fig. 12, BECCS provides significantly greater value to the electricity system than DAC, though this gap does narrow substantially once more than $4 \mathrm{GW}$ of capacity are installed.

\subsection{Value Transfer}

In section 3, we showed that the availability of both BECCS and DACS increased the utilisation of CCGT 397 power plants. In the absence of NETs, they would have been constrained off the system owing to their $\mathrm{CO}_{2} 398$

\footnotetext{
IEfficiency of CCGT-CCS power plant
} 


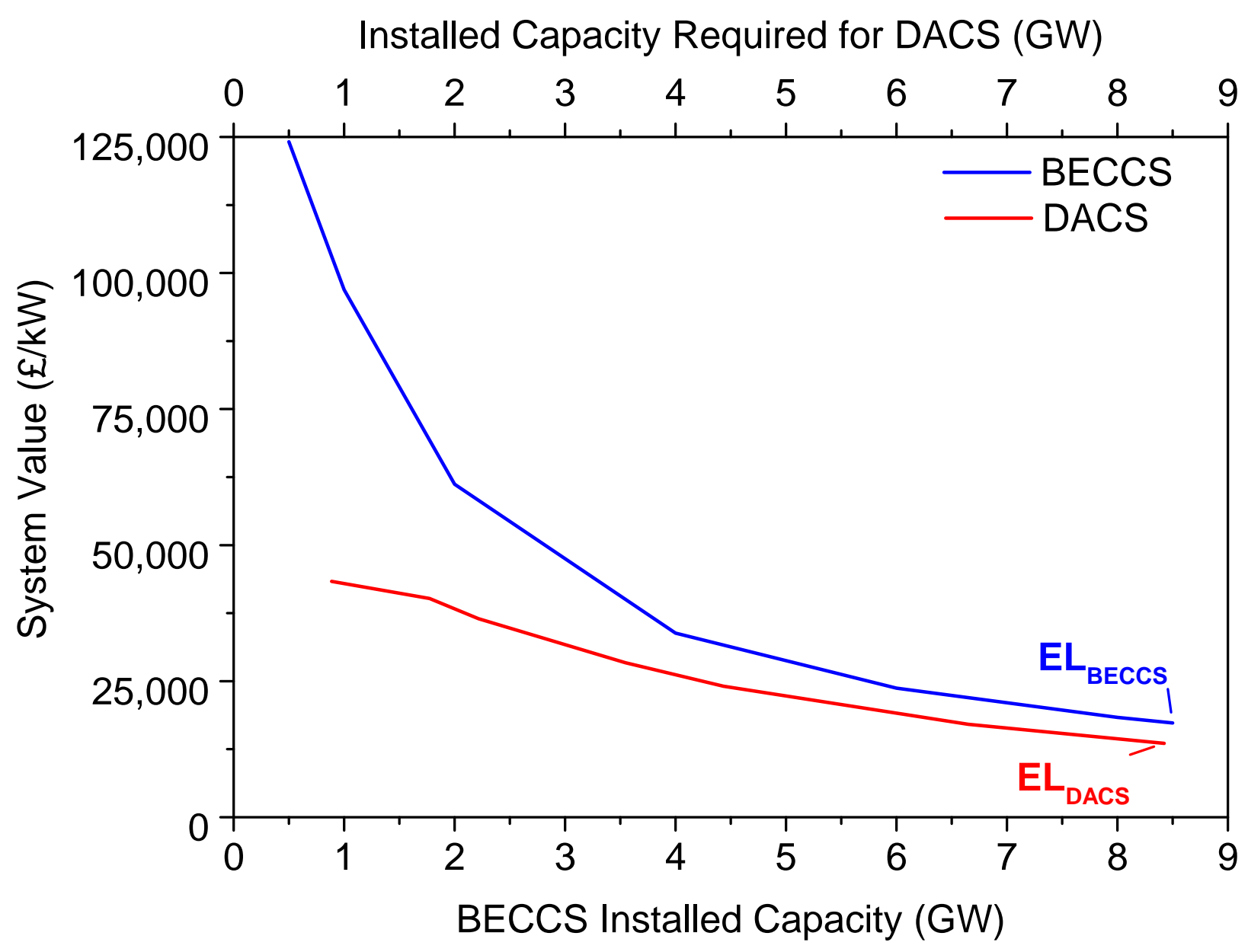

Figure 12: System value of BECCS and DACS to the UK electricity system. Their economic levels (EL) of deployment are highlighted.

emissions. Fig. 13 illustrates the contributions of different technologies to power sector emissions from 399 2015 to 2050 when NETs are available to the system.

It is observed that, with increasing availability of NETs, the asset utilisation of CCGTs is also substantially 401 increased, whilst only marginal increases are observed for interconnection, OCGT and CCGT-CCS assets. 402 In other words, the availability of NETs allows for increased revenue to be earned by those generation assets 403 that would be otherwise constrained off the electricity grid owing to their carbon emissions. In order to fairly 404 distribute the cost of providing this service, it is essential to allow some of that value to accrue to the NET 405 facilities that are providing this service, thus reducing the societal cost of delivering negative emissions. In 406 this section, we propose a proportional value transfer mechanism, and illustrate how it might be calculated. 407

Fig. 14 shows how CCGT utilisation factor $\left(\mathrm{UF}_{\mathrm{CCGT}}\right)$ vary with different availabilities of BECCS 408 technology. It is evident that higher $\mathrm{UF}_{\mathrm{CCGT}}$ are associated with BECCS deployment. In the period 409

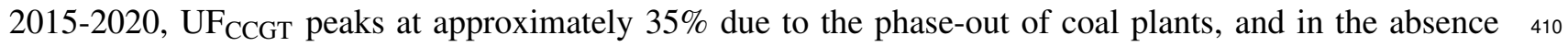

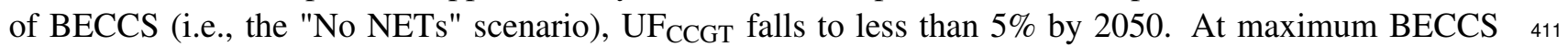
deployment, however, $\mathrm{UF}_{\mathrm{CCGT}}$ is seen to be consistently higher than current levels, reaching $49 \%$ by 2050 . ${ }^{412}$ To quantify the value of the emissions offset provided by BECCS to CCGT, a Value Transfer metric is used. $\quad{ }^{413}$ 


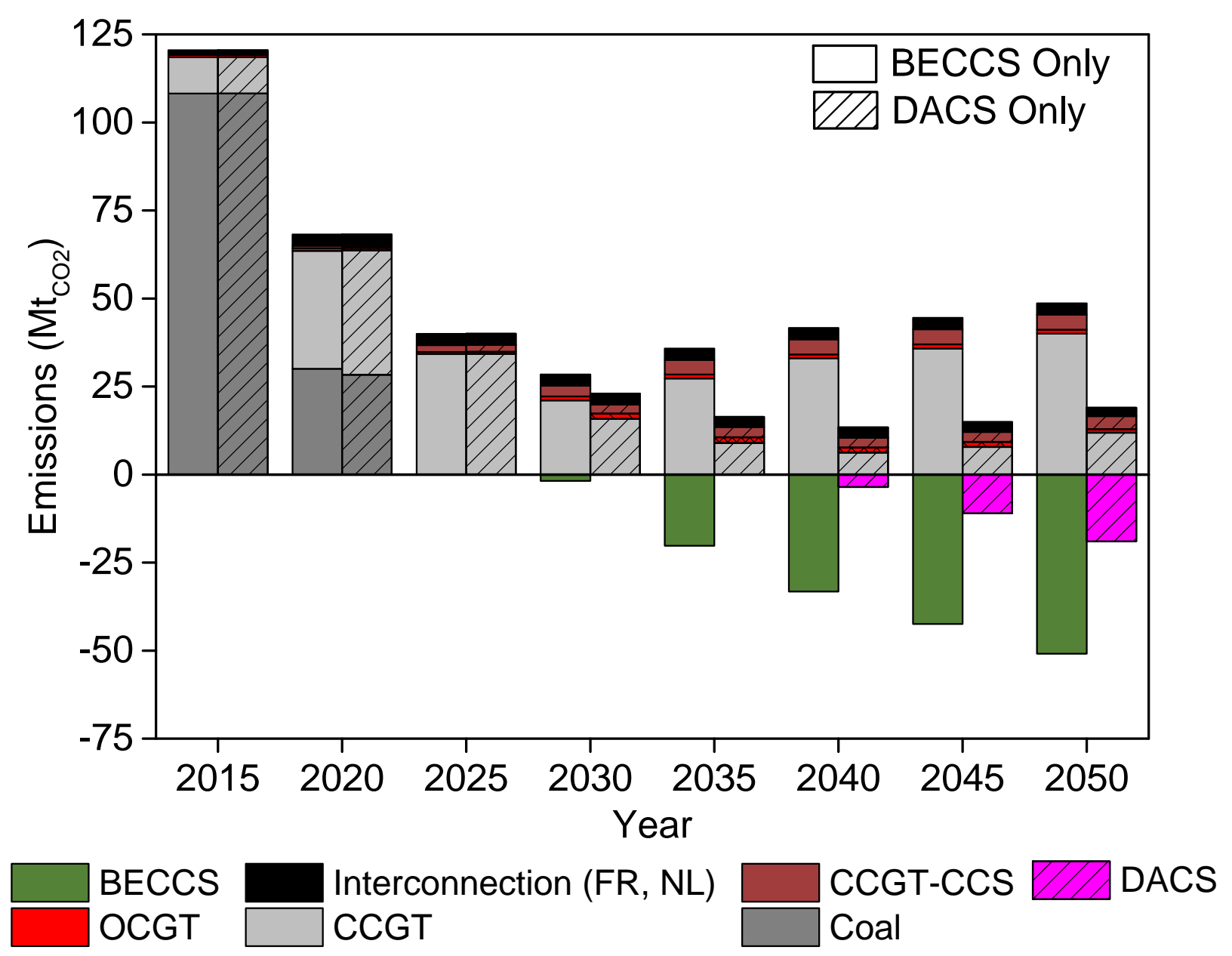

Figure 13: Carbon emissions by technology in a UK electricity system that achieves complete decarbonisation by 2050 .

The Value Transfer $\left(\mathrm{VT}_{\mathrm{i}, \mathrm{j}}\right)$ metric, presented in Eq. 4, quantifies the value transferred to technology $j$ by the 414 increased operation of negative emissions technology $i$ at deployment level $d, N E_{i, d}$. It has been defined as 415 the change in revenue generated by technology $j$ divided by the change in negative emissions provided by 416 technology $i$.

$$
V T_{\mathrm{i}, \mathrm{j}}=\frac{R_{j, d}-R_{j, \text { initial }}}{N E_{i, d}}
$$

where: $V T$ is the value added to technology $j$ plants by increased deployment of $i ; R_{j, d}$ is the total revenue 418 generated by $j$ from 2015 to 2050 when $i$ is deployed at capacity $d ; R_{j, \text { initial }}$ is the total revenue generated by $j$ when no negative emissions technologies are deployed in the system; and $N E_{i, d}$ is the total negative emissions provided by $i$ at capacity $d$.

Revenue generated by $j, R_{j}$ has been calculated as the sum of its revenue from electricity generation to meet 422 demand and revenue from provision of reserve capacity. This is shown in the equation below:

$$
R_{j}=\sum_{a, t} p 2 d_{j, a, t} \times M C_{a, t}+\sum_{a, t} r_{j, a, t} \times M R P_{a, t}
$$

where $t$ is time, discretised hourly; $a$ is the year in which $j$ is providing that service to demand; $p 2 d_{j, a, t}{ }_{424}$ is the electricity generated hourly by technology $j$ to meet demand at time $t$ in year $a(\mathrm{MWh} / \mathrm{hr}) ; M C_{a, t}$ is ${ }_{425}$ 


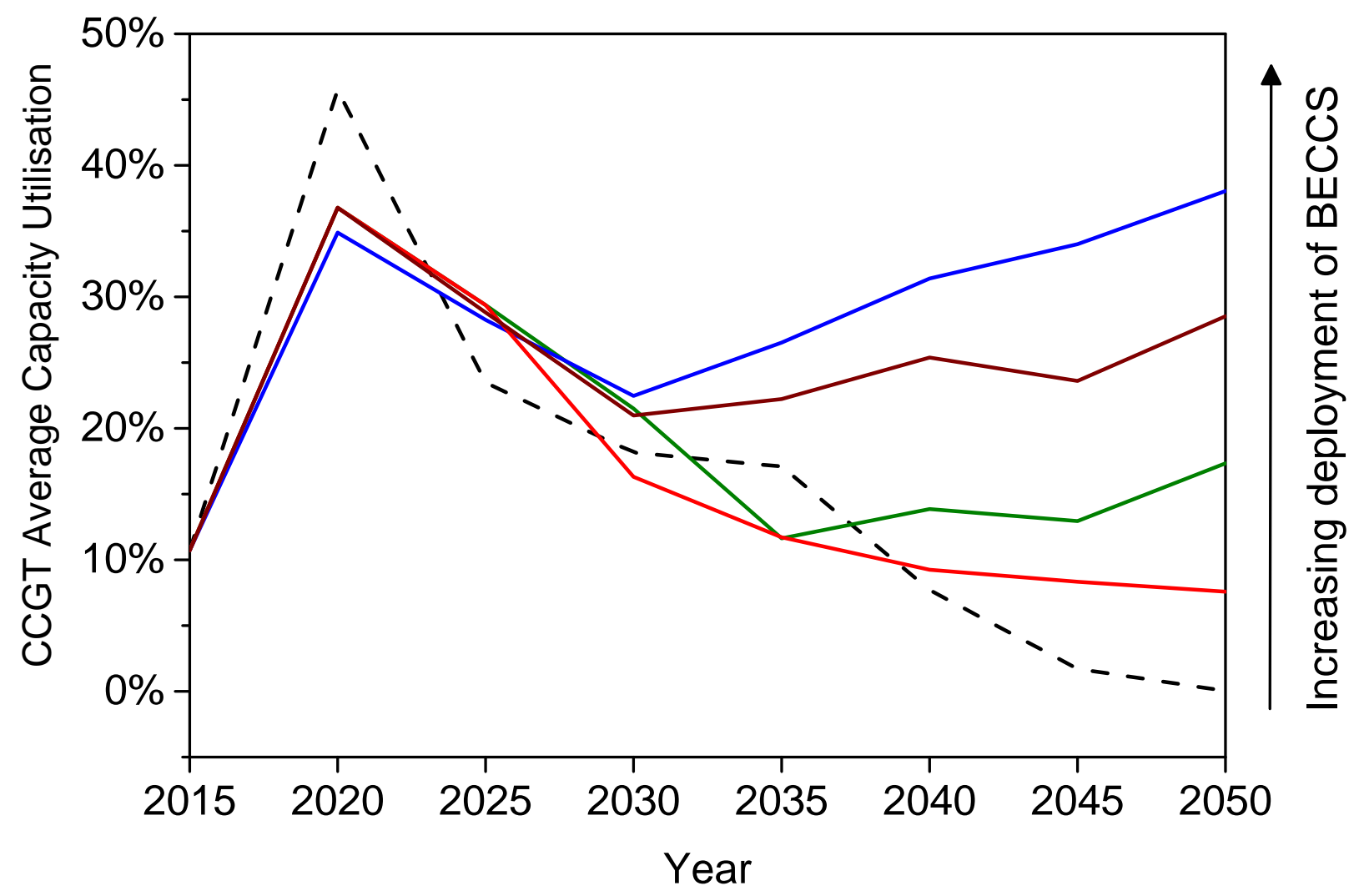

\section{BECCS Availability}

--- No NETs $-2 \mathrm{GW}-4 \mathrm{GW}-6 \mathrm{GW}-8.5 \mathrm{GW}$

Figure 14: Variation in the utilisation factor of CCGT power plants with increased BECCS deployment in the electricity system.

the marginal cost of electricity ( $\left(\right.$ /MWh) at time $t$ in year $a$, derived as explained in section $2.1, r_{j, a, t}$ is the 426 reserve provided by $j$ at time $t$ in year $a(\mathrm{MWh})$; and $M R P_{a, t}$ is the marginal cost of reserve at time $t$ in year ${ }_{427}$ $a(\mathfrak{E} / \mathrm{MWh})$.

Fig. 15 shows the variation in CCGT revenues and negative emissions provided with installed capacity 429 of BECCS and DACS. $V T_{D A C S O n l y}$ and $V T_{B E C C S O n l y}$ were calculated to be approximately $£ 460 / \mathrm{t}_{\mathrm{CO}_{2}}$ and 430 $£ 230 / \mathrm{t}_{2}$. Therefore, per tonne of $\mathrm{CO}_{2}$ removal, DACS accrues twice the value to CCGTs that BECCS ${ }_{431}$ does. From 2015 to $2050, £ 35$ billion and $£ 16$ billion of additional revenue was generated by CCGTs 432 when BECCS and DACS are deployed to their economic limit, respectively. $V T_{D A C S}$ is greater due to ${ }_{433}$ the higher MCs (illustrated in Fig. 10) and MRPs when DACS is deployed in the electricity system. In 434 addition, BECCS ability to provide reserve reduces the need for CCGT reserve in the system. In a DACS 435 Only scenario, CCGT reserve provision and MRP peak at $70 \%$ and $£ 43 / \mathrm{MWh}$, respectively, compared to 436 $59 \%$ and $£ 22 / \mathrm{MWh}$, respectively, in a BECCS Only scenario. This results in larger CCGT revenues despite ${ }_{437}$ reduced operation (see Fig. 8). BECCS ability to provide reserve capacity in the system therefore reduces 438 revenues generated by CCGTs. 

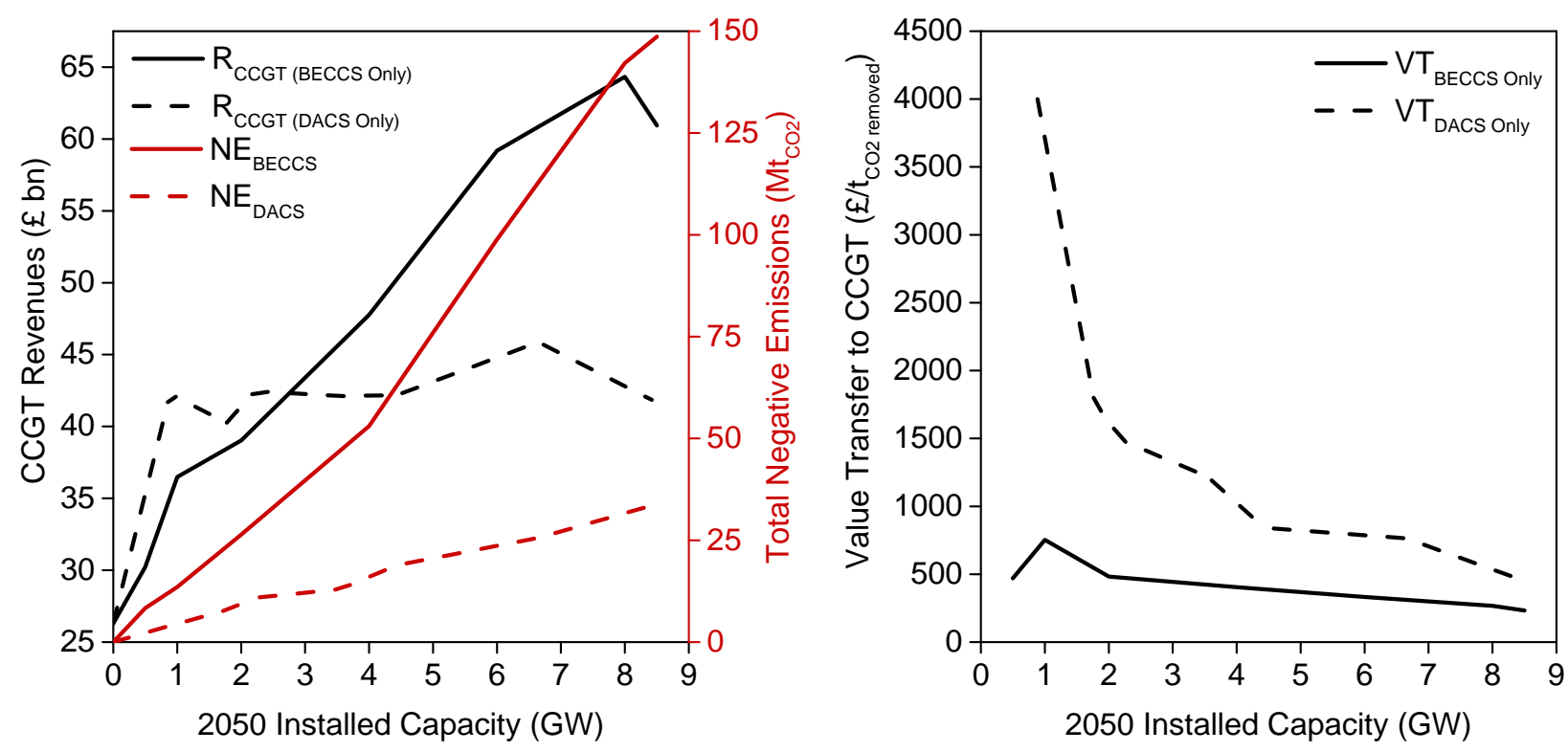

Figure 15: Variation in the total revenues generated by CCGT power plants and negative emissions provided with increasing deployment of BECCS and DACS (left). Value transfer from BECCS and DACS to CCGT power plants at different installed capacities (right).

\subsection{Operation of BECCS within the electricity system}

BECCS has been shown to provide three distinct services to the electricity system: power generation to meet demand, $\mathrm{CO}_{2}$ removal from the atmosphere, and value transferred to $\mathrm{CO}_{2}$ emitting assets that would be otherwise constrained off the electricity grid. As the value of, and thus appropriate compensation for, each service is likely to differ in value, it is important to understand the role of BECCS in the electricity system in which it is deployed. BECCS operation, described by its utilisation factor, $U F_{B E C C S}$, is investigated to understand this role.

Fig. 16 shows the average hourly $U F_{B E C C S}$ and $U F_{C C G T}$ in 2030 (when deployment begins) and 2050 (when decarbonisation is to be achieved) compared to the average annual hourly demand. In 2030, BECCS power generation is seen to fluctuate with hourly demand, i.e. it is load-following. CCGTs are observed to exhibit the same behaviour. BECCS is therefore operating to meet some electricity demand and provide some offset for CCGT emissions. The levelised cost of BECCS electricity is calculated to be $£ 234 / \mathrm{MWh}$ (£64/MWh is contributed by biomass costs alone). Alternatively, the levelised $\mathrm{CO}_{2}$ abatement cost (total investment in BECCS per tonne of $\mathrm{CO}_{2}$ removed) is approximately $£ 185 / \mathrm{t}_{\mathrm{CO}_{2}}$. These do not included the value it should accrue by providing a service to CCGT, discussed in section 4. In 2050, however, $U F_{B E C C S}$ is consistently at its maximum of $85 \%$, hence it is operating base load. Therefore as the system emissions target tends to zero, BECCS transitions from load-following to base load behaviour, with the latter allowing for maximum $\mathrm{CO}_{2}$ removal to meet the decarbonisation objective.

There is currently no incentive to support the deployment of NETs. The above analysis has, however, 458 described the potential value that can be added by BECCS and DACS to the electricity system as a whole. In practice, this added value should accrue to the NETs in the form of a negative emissions credit, as discussed in previous work ${ }^{78}$. This has not been considered in this study.

Although the services to the electricity system provided by BECCS (electricity generation, reserve provision 462 and negative emissions) and DACS (negative emissions) differ, they can both provide emissions offset that 


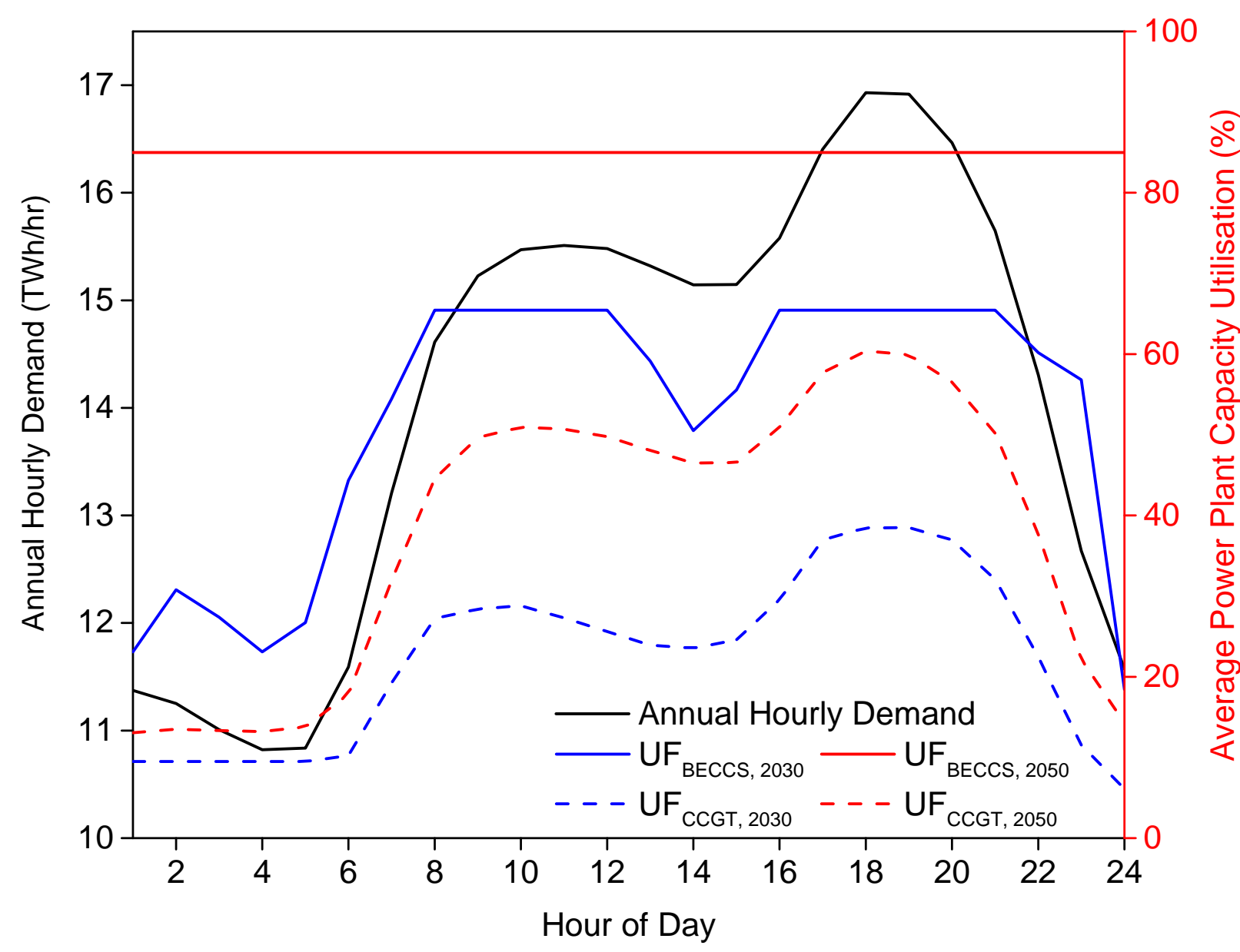

Figure 16: Variation in the average utilisation factor of BECCS plants in 2030 and 2050 with hourly electricity demand. The load factors are to be read of the right vertical axis (red), while the annual hourly demand is given on the left vertical axis (black).

allows for continued operation of cheaper unabated and abated thermal plants, especially CCGTs, and 464 consequently drive down system cost. The Value Transfer metric, $V T_{i, j}$, discussed in section 4.2 shows 465 that this continued utilisation translates into larger revenues for $\mathrm{CO}_{2}$ emitting assets. The compensation of 466 NETs by carbon emitters (at a price of $V T_{i, j}$ ) can potentially reduce the public burden of delivering negative ${ }_{467}$ emissions, i.e. reduce the negative emissions credit that needs to be provided for NETs' 'social service' of 468 $\mathrm{CO}_{2}$ removal.

\section{Sensitivity analysis}

The scenarios discussed in section 3 are dependent on the availability of CCGT-CCS technology, which is not currently deployed. In addition, estimates for BECCS and DACS technology costs have been used in the analysis. The influence of varying CCGT-CCS availability and NETs costs on the need for, and source of, negative emissions was investigated and is discussed below. 


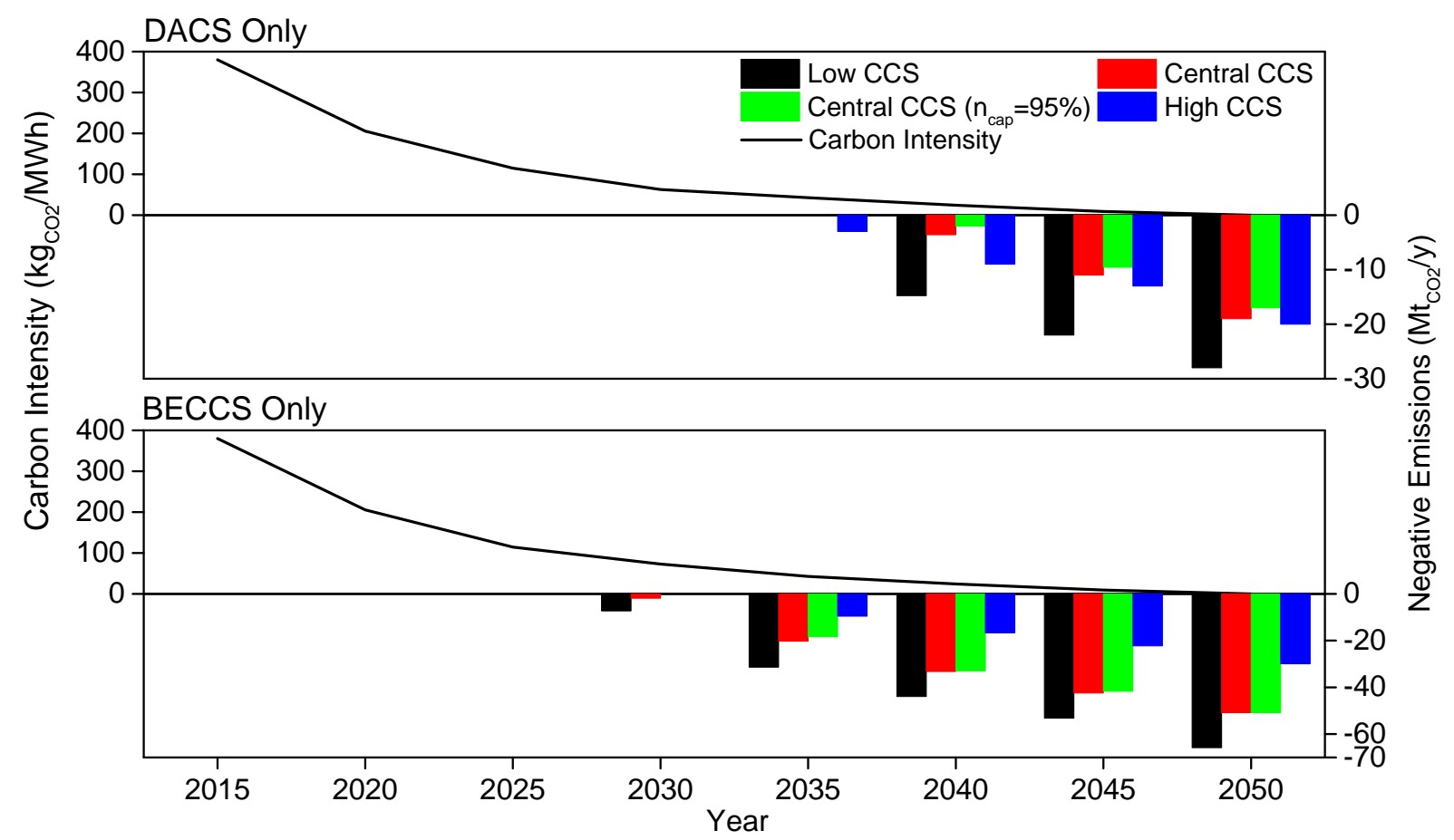

Figure 17: The variation in the amount of negative emissions deployed in the system from 2015-2050 with different CCGT-CCS availabilities, subject to a zero emissions target for the electricity system in 2050. Note that the vertical axis (negative emissions provided) has different scales for the DACS Only and BECCS Only scenarios.

\subsection{CCS availability}

CCGT-CCS contributes $15 \mathrm{GW}$ to installed capacity in all the scenarios that achieve complete 476 decarbonisation by 2050. It has been argued that the deployment of CCS technology presents a moral hazard to climate change mitigation as it allows for the continued consumption of fossil fuels ${ }^{80 \mid 81}$. It has also been shown that CCS can achieve capture rates of $>90 \%$, which is conventionally assumed, with limited efficiency penalty ${ }^{70 \mid 82}$. The amount of BECCS or DACS needed to decarbonise by 2050 at different CCGT-CCS availabilities and capture rates was investigated. Four scenarios are illustrated in Fig. 17; a) Low CCS (5 GW), b) Central CCS (15 GW), c) High CCS (45 GW), and d) Central CCS with $\eta_{\text {capture }}=95 \%$.

In the DACS Only scenario, increased CCGT-CCS availability initially reduces the need for negative emissions. However, at CCGT-CCS capacity of $>15 \mathrm{GW}$ (Central CCS), more $\mathrm{CO}_{2}$ removal is needed to compensate for rising residual CCS emissions. Fig. 17] shows that DACS removes $1 \mathrm{Mt}_{\mathrm{CO}_{2}} / \mathrm{y}$ more in a High CCS scenario, compared to a Central CCS scenario. This is because increased CCGT-CCS operation largely displaces Nuclear from the system, although CCGT generation is also reduced. As CCGT-CCS capacity increases from 5 to $45 \mathrm{GW}$, added Nuclear capacity decreases from $24 \mathrm{GW}$ to $1.2 \mathrm{GW}$. In 2050, electricity demand met by Nuclear falls from 167 to $8 \mathrm{TWh} / \mathrm{y}$; CCGT service to demand also falls from 66 to $16 \mathrm{TWh} / \mathrm{y}$. Overall, $\mathrm{CO}_{2}$ removal by DACS falls from 28 to $20 \mathrm{Mt}_{\mathrm{CO}_{2}} / \mathrm{y}$, from low to high CCS availability. Therefore, from a systems perspective, it is cheaper to operate more abated gas plants with DACS compensating for their residual emissions than to have a Nuclear-dominated electricity system. Furthermore, even at very high CCS availabilities, NETs are deployed in the optimal electricity system. 
increases from $5 \mathrm{GW}$ to $45 \mathrm{GW}$, negative emissions needed reduces from $66 \mathrm{Mt}_{\mathrm{CO}_{2}} / \mathrm{y}$ to $30 \mathrm{Mt}_{\mathrm{CO}_{2}} / \mathrm{y}$ in 495 2050. This is because increased CCGT-CCS generation displaces CCGT generation from the system - as 496 CCGT-CCS capacity increases, CCGT generation falls from 164 to $42 \mathrm{TWh} / \mathrm{y}$ in 2050 . Less emissions 497 resulting from CCGT outweigh the increased residual emissions from CCGT-CCS, hence the need for 498 negative emissions to compensate is reduced.

Increased capture rate, however, is found to have much less influence as a 5\% increase in capture rate reduces 500 the need for negative emissions by approximately $2 \mathrm{Mt}_{\mathrm{CO}_{2}} / \mathrm{y}$ in the DACS Only scenario. In the BECCS 501 Only scenario, the change is negligible. This occurs as reduced CCS emissions do not increase CCGT-CCS 502 utilisation, rather cheaper CCGT plants produce $6 \mathrm{TWh} / \mathrm{y}$ more. The resulting CCGT emissions cancel out 503 reduced CCS emissions, hence the need for $\mathrm{CO}_{2}$ removal remains the same.

\subsection{Scope for Cost Reduction}

Thus far, we have evaluated the system with only one of BECCS or DACS available in any given scenario. However, given the relative immaturity of both technologies, and the potential for cost reduction, it is instructive to evaluate how they would interact in an electricity system if they were both available and competing. Thus, in this section, we present a sensitivity analysis quantifying the extent to which each technology would contribute to meeting a given negative emissions target should both technologies be available over a wide range of capital and operational costs.

\subsubsection{CAPEX}

Cost estimates in literature for DACS technology are for first-of-a-kind plants 23 [24|55! This is likely to reduce with increased R\&D and deployment. The development of modular units for DACS ${ }^{22 \mid 23}$ may also benefit from economies of scale, through mass production. Using the costs provided in Table 1 , it was found that $87 \%$ of the investment in DACS was dedicated to CAPEX, with 17\% for OPEX (Fig. 18). In contrast, investment in BECCS was made up of $60 \%$ CAPEX and $40 \%$ OPEX.

Fig. 19 shows the contribution of DACS to the UK's Paris Agreement target at different BECCS and 518 DACS CAPEX. At current BECCS costs, CAPEX ${ }_{\text {DACS }}$ must fall by a factor of 10 before its deployed 519 for negative emissions. If the capital cost of DACS is reduced below $3500 / \mathrm{kW}_{\mathrm{e}}$. DACS contributes $1-13 \%$ of $-50 \mathrm{Mt}_{\mathrm{CO}_{2}} / \mathrm{y}$ target in 2050 (Fig. 2). At current cost estimates however, DACS remains uncompetitive with BECCS technology, even if CAPEX ${ }_{\text {BECCS }}$ increases more than twofold from estimates in section 2.1.

506 507 508 509

\subsubsection{Biomass cost}

523

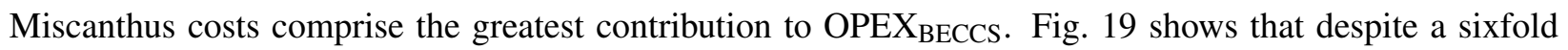
increase in Miscanthus raw material costs, BECCS is still the preferred source of all negative emissions. In addition to this increase, $\mathrm{CAPEX}_{\mathrm{BECCS}}$ must be $>4000 / \mathrm{kW}$ (1.5 times the current CAPEX) for DACS to contribute to meeting negative emissions targets.

\section{Conclusions}

This study finds that, while power sector decarbonisation by 2050 is theoretically achievable without 529 negative emissions technologies (NETs), this would require significant expansion of capital-intensive

"Note that this is the capital costs of DACS levelised against its electricity consumption only, as discussed in section 2.1 


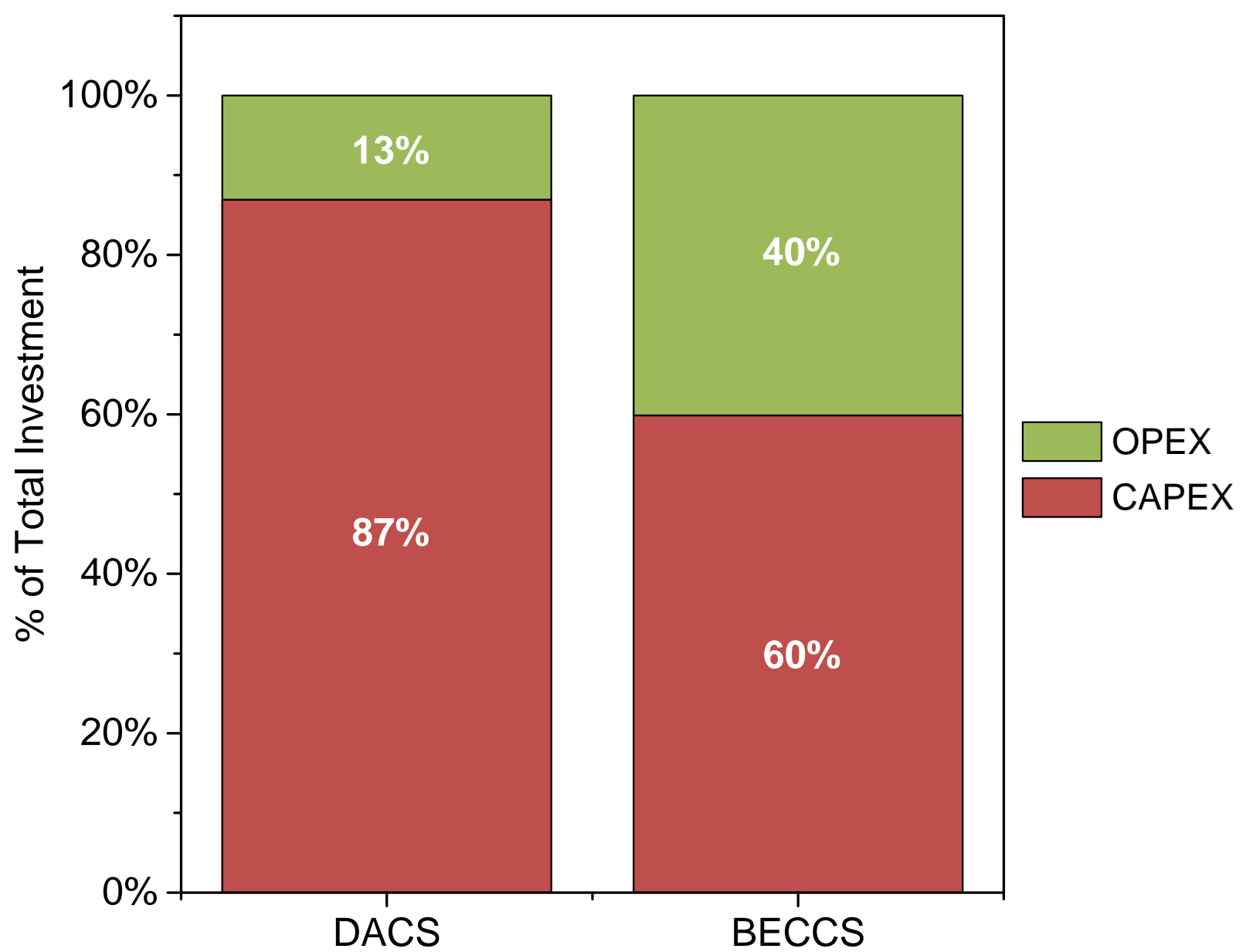

Figure 18: Contribution of CAPEX and OPEX to the total investment required for the operation of BECCS and DACS in the BECCS Only and DACS Only scenarios, respectively.

intermittent renewables and nuclear technologies. This results in a fivefold increase in electricity costs by 531 2050. Public acceptability is likely to hinder such a transition due to the lack of affordability of electricity. In 532 addition, there are further sociopolitical barriers that must be considered. The large penetration of land-based 533 IRES will require land dedicated to solar and onshore wind farms. Issues arising due to competition for land 534 and social attitudes ${ }^{77}$ must be overcome. Lastly, conventional nuclear technology faces challenges of large 535 financing costs and long payback periods (discussed in section 1.1), which may dissuade from the pursuit of 536 further projects ${ }^{43 / 44}$. Should NETs not be considered, it is unlikely for the decarbonisation target to be met. 537

Despite their relative costliness, NETs deployment could reduce total investment needed for electricity 538 generation by $37-48 \%$ as their negative emissions allow for increased utilisation of cheaper $\mathrm{CO}_{2}$ emitting 539 generators within the system. BECCS provides the greatest opportunity for cost reduction. It is found to 540 provide three times greater reduction in total system cost of the electricity system than DACS, per installed 541 capacity. Whilst DACS still adds value to the system, its deployment will drive up electricity prices to levels 542 seen in a No NETs system due to its large operating costs. A significant ramping of nuclear capacity installed 543 is also needed. In contrast, BECCS deployment displaces the need for new nuclear plants as the services it 544 provides - flexible carbon-negative generation to meet demand, reserve, and enable the continued utilisation 545 of cheaper gas plants - are more valuable than the base load low-carbon generation offered by Nuclear. 546 

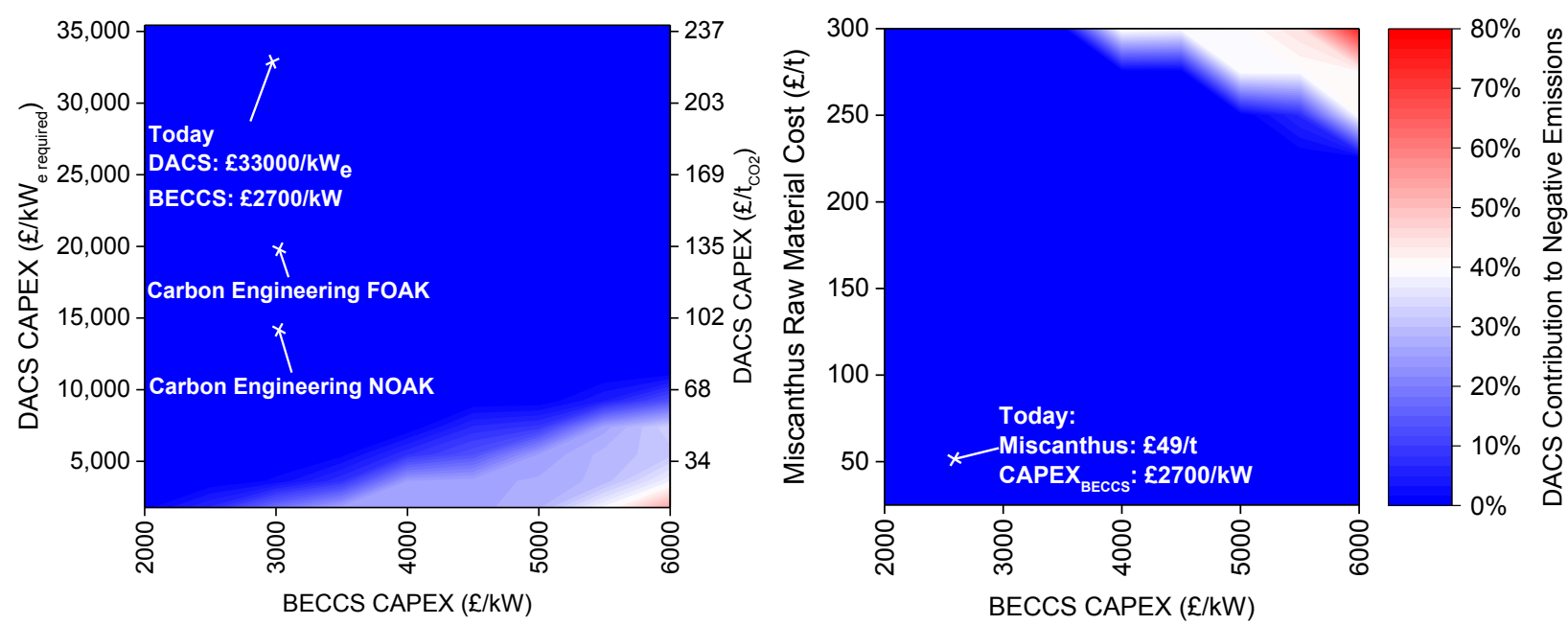

Figure 19: Contribution of DACS to the UK's negative emissions target at different biomass, BECCS and DACS costs.

Local grassland yield and availability is sufficient to satisfy the biomass demand for decarbonisation by 547 2050. However for the deeper decarbonisation pathways needed to be compliant with the Paris Agreement, 548 $7 \%$ of biomass demand needs to be imported. The more stringent targets will also increase total electricity system cost by at least $10 \%$ (when BECCS is deployed).

The emissions offset that negative emissions provide for $\mathrm{CO}_{2}$ emitting generators, especially CCGTs, has been observed to translate to larger revenues for CCGTs. In the absence of an incentive for negative emissions, a value transfer mechanism between NETs and $\mathrm{CO}_{2}$ emitting assets could serve to reduce the public-borne cost of delivering negative emissions. This study concludes that NETs significantly reduce the cost of decarbonisation of the UK electricity system by 2050, and compensation by $\mathrm{CO}_{2}$ emitting assets could offer a potential route to commercial deployment.

\section{Declaration of interest}

Declarations of interest: none.

\section{Acknowledgements}

The authors thank the "Science and Solutions for a Changing Planet Doctoral Training Programme" (SSCP 560 DTP) by the Natural Environment Research Council (NERC), the IEA Greenhouse Gas R\&D Programme ${ }_{561}$ (IEAGHG), the MESMERISE-CCS project under grant EP/M001369/1 from the Engineering and Physical 562 Sciences Research Council (EPSRC), and the "Comparative assessment and region-specific optimisation of 563 GGR" project under grant NE/P019900/1 from NERC for the funding of PhD scholarships and support of 564 this project.

\section{References}

[1] M. R. Allen, D. J. Frame, C. Huntingford, C. D. Jones, J. A. Lowe, M. Meinshausen, and 567 N. Meinshausen, "Warming caused by cumulative carbon emissions towards the trillionth tonne," 568 
Nature, vol. 458, pp. 1163-1166, Apr. 2009.

[2] M. Meinshausen, N. Meinshausen, W. Hare, S. C. B. Raper, K. Frieler, R. Knutti, D. J. Frame, and 570 M. R. Allen, "Greenhouse-gas emission targets for limiting global warming to $2^{\circ} \mathrm{C}$, , Nature, vol. $458, \quad 571$ pp. 1158-1162, Apr. 2009.

[3] IPCC, Climate Change 2013: The Physical Science Basis. Contribution of Working Group I to the 573 Fifth Assessment Report of the Intergovernmental Panel on Climate Change [T. F. Stocker and D. Qin 574 and G.-K. Plattner and M. Tignor and S. K. Allen and J. Boschung and A. Nauels and Y. Xia and 575 V. Bex and P. M. Midgley (eds.)]. Cambridge, United Kingdom: Cambridge University Press, 2009. 576 $1535 \mathrm{pp}$.

[4] J. C. Minx, W. F. Lamb, M. W. Callaghan, L. Bornmann, and S. Fuss, "Fast growing research on 57 negative emissions," Environ. Res. Lett., vol. 12, p. 035007, Mar. 2017.

[5] IPCC, Climate Change 2014: Mitigation of Climate Change. Contribution of Working Group III to 580 the Fifth Assessment Report of the Intergovernmental Panel on Climate Change [O. Edenhofer and 58 R. Pichs-Madruga and Y. Sokona and E. Farahani and S. Kadner and K. Seyboth and A. Adler and 582 I. Baum and S. Brunner and P. Eickemeier and B. Kriemann and J. Savolainen and S. Schlömer and 583 C. von Stechow and T. Zwickel and J. C. Minx (eds.)]. Cambridge, United Kingdom: Cambridge 584 University Press, 2014.

[6] J. K. Stolaroff, D. W. Keith, and G. V. Lowry, "Carbon dioxide capture from atmospheric air using 586 sodium hydroxide spray,” Environmental Science \& Technology, vol. 42, pp. 2728-2735, Apr. $2008 . \quad 587$

[7] R. Baciocchi, G. Storti, and M. Mazzotti, "Process design and energy requirements for the capture 588 of carbon dioxide from air," Chem. Eng. Process. Process Intensif., vol. 45, no. 12, pp. 1047-1058, 589 2006.

[8] G. Lomax, T. M. Lenton, A. Adeosun, and M. Workman, "Investing in negative emissions," Nat. 591 Clim. Change, vol. 5, pp. 498-500, May 2015.

[9] R. S. Lampitt, E. P. Achterberg, T. R. Anderson, J. A. Hughes, M. D. Iglesias-Rodriguez, B. A. 593 Kelly-Gerreyn, M. Lucas, E. E. Popova, R. Sanders, J. G. Shepherd, D. Smythe-Wright, and A. Yool, 594 "Ocean fertilization: a potential means of geoengineering?," Philosophical Transactions of the Royal 595 Society A: Mathematical, Physical and Engineering Sciences, vol. 366, pp. 3919-3945, Nov. 2008.

[10] P. Renforth and T. Kruger, "Coupling mineral carbonation and ocean liming," Energy \& Fuels, 597 vol. 27, pp. 4199-4207, Aug. 2013.

[11] T. M. Lenton and C. Britton, "Enhanced carbonate and silicate weathering accelerates recovery from fossil fuel $\mathrm{CO}_{2}$ perturbations," Global Biogeochem. Cycles, vol. 20, pp. 1-12, Aug. 2006.

[12] R. D. Schuiling and P. Krijgsman, "Enhanced Weathering: An Effective and Cheap Tool to Sequester $\mathrm{CO}_{2}$," Clim. Change, vol. 74, pp. 349-354, Jan. 2006.

[13] P. Renforth, D. A. C. Manning, and E. Lopez-Capel, "Carbonate precipitation in artificial soils as a sink for atmospheric carbon dioxide," Appl. Geochem., vol. 24, pp. 1757-1764, Sept. 2009.

[14] N. R. McGlashan, M. H. W. Workman, B. Caldecott, and N. Shah, "Negative Emissions 605 Technologies." Grantham Institute for Climate Change Briefing paper No 8, Oct. 2012. 
[15] United Nations Framework Convention on Climate Change, "Adoption of the Paris Agreement," Dec. 607 2015.

[16] Great Britain, “Climate Change Act 2008: Elizabeth II.” Stationery Office, London, 2008.

[17] Committee on Climate Change, "Power sector scenarios for the fifth carbon budget." http: 610 //tinyurl.com/ycb58x74, 2015.

[18] Committee on Climate Change, "UK climate action following the Paris Agreement," Oct. 2016.

[19] A. Vina, W. J. McConnell, H. Yang, Z. Xu, and J. Liu, "Effects of conservation policy on chinas 613 forest recovery," Science Advances, vol. 2, pp. e1500965-e1500965, Mar. 2016.

[20] World Resources Institute, "Initiative 20x20: Bringing 20 million hectares of degraded land in Latin 615 America and the Caribbean into restoration by 2020.." http://www.wri.org/our-work/ 616 project/initiative-20x20/about-initiative-20x20. Accessed on 14/12/2017., 617 Dec. 2017.

[21] Sallie Greenberg (Ed.), "Illinois Basin - Decatur Project," Greenhouse Gases Sci. Technol., vol. 4, 619 pp. 569-570, Sept. 2014. Special Issue.

[22] Carbon Engineering Ltd, “Our Technology,” Apr. 2015.

[23] Climeworks AG, “Climeworks $\mathrm{CO}_{2}$ Capture Demonstrator,” Apr. 2016.

[24] Global Thermostat Ltd, “About Us: A Unique Capture Process,” 2010.

[25] P. Smith, S. J. Davis, F. Creutzig, S. Fuss, J. Minx, B. Gabrielle, E. Kato, R. B. Jackson, A. Cowie, 624 E. Kriegler, D. P. van Vuuren, J. Rogelj, P. Ciais, J. Milne, J. G. Canadell, D. McCollum, G. Peters, 625 R. Andrew, V. Krey, G. Shrestha, P. Friedlingstein, T. Gasser, A. Grübler, W. K. Heidug, M. Jonas, 626 C. D. Jones, F. Kraxner, E. Littleton, J. Lowe, J. R. Moreira, N. Nakicenovic, M. Obersteiner, 627 A. Patwardhan, M. Rogner, E. Rubin, A. Sharifi, A. Torvanger, Y. Yamagata, J. Edmonds, and 628 C. Yongsung, "Biophysical and economic limits to negative $\mathrm{CO}_{2}$ emissions," Nat. Clim. Change, vol. 6, pp. 42-50, Dec. 2015.

[26] Department for Trade and Industry, "Meeting the Energy Challenge. A White Paper on Energy," May 631 2007.

[27] UK Department of Energy \& Climate Change, "The UK low carbon transition plan: national strategy 633 for climate and energy.” Policy paper, July 2009.

[28] Department for Business, Energy \& Industrial Strategy, "Implementing the End of Unabated Coal by 2025. Government response to unabated coal closure consultation,” Jan. 2018.

[29] Department for Business, Energy and Industrial Strategy (BEIS), "Capacity of, and electricity ${ }_{637}$ generated from, renewable sources (DUKES 6.4).” Renewables statistics, Digest of UK Energy 638 Statistics (DUKES), and Energy and climate change: evidence and analysis, July 2017.

[30] Great Britain, “Energy Act 2008: Elizabeth II.” Stationery Office, London, Nov. 2008.

[ [31] Office of Gas and Electricity Markets, “About the FIT scheme." https://www. ofgem.gov • 641 uk/environmental-programmes/fit/about-fit-scheme, 2017. 
[32] European Union, "Directive 2009/28/EC of the European Parliament and of the Council of 23 April 643 2009 on the promotion of the use of energy from renewable sources and amending and subsequently 644 repealing Directives 2001/77/EC and 2003/30/EC.” Office Journal of the European Union, June 2009. 645

[33] Great Britain, “Chapter 32. Energy Act 2013: Elizabeth II,” Dec. 2013.

[34] Office of Gas and Electricity Markets, "Electricity interconnectors." https : / /www. of gem.gov . 647 uk/electricity/transmission-networks/electricity-interconnectors, $\quad 648$ 2017.

[35] Department for Business, Energy \& Industrial Strategy (BEIS), "Historical electricity data: 1920 to 650 2016.” Electricity statistics, July 2017.

[36] P. Heptonstall, R. Gross, and F. Steiner, "The costs and impacts of intermittency - 2016 update," Feb. 652 2017.

[37] Y. Yasuda, L. Bird, E. M. Carlini, A. Estanqueiro, D. Flynn, A. Forcione, E. Gómez-Lázaro, 654 P. Higgins, H. Holttinen, D. Lew, S. Martin-Martinez, J. McCann, N. Menemenlis, and J. C. Smith, 655 "International Comparison of Wind and Solar Curtailment Ratio," in Proceedings of WIW2015 Workshop, Oct. 2015.

[38] L. Bird, D. Lew, M. Milligan, E. M. Carlini, A. Estanquiero, D. Flynn, E. Gómez-Lázaro, 658 H. Holtinnen, N. Menemenlis, A. Orths, P. B. Eriksen, J. C. Smith, L. Soder, P. Sorensun, 659 A. Altiparmakis, Y. Yasuda, and J. Miller, "Wind and solar energy curtailment: A review of 660 international experience," Renewable Sustainable Energy Rev., vol. 65, pp. 577-586, 2016.

[39] National Grid, “Monthly Balancing Services Summary 2016.” http: 662 //www2.nationalgrid.com/UK/Industry-information/ 663 Electricity-transmission-operational-data/Report-explorer/ 664 Services-Reports/. Accessed: 04-07-2017, 2016.

[40] Renewable Energy Foundation (REF), "Wind Farm Constraint Payments over Easter 2016," Mar. 666 2016.

[41] Institution of Civil Engineers, "Electricity Storage: Realising the Potential." 668 https://www.ice.org.uk/getattachment/media-and-policy/policy/ 669 electricity-storage-realising-the-potential/ICE-(2015) -Electricity-Storage-Realising-the-Potential.pdf.aspx, 2015.

[42] A. Morse, "Nuclear power in the UK." National Audit Office, July 2016.

[43] A. Vaughan, "Hinkley Point C subsidy has dealt consumers 'a bad hand', say MPs," The Guardian, 673 Nov. 2017.

[44] A. Morse, "Hinkley Point C.” National Audit Office, June 2017.

[45] E. S. Rubin, I. M. L. Azevedo, P. Jaramillo, and S. Yeh, "A review of learning rates for electricity supply technologies," Energy Policy, vol. 86, pp. 198-218, 2015.

[46] J. R. Lovering, A. Yip, and T. Nordhaus, "Historical construction costs of global nuclear power 678 reactors," Energy Policy, vol. 91, pp. 371-382, Apr. 2016. 
[47] M. Fajardy and N. Mac Dowell, "Can BECCS deliver sustainable and resource efficient negative 680 emissions?," Energy \& Environmental Science, vol. 10, no. 6, pp. 1389-1426, 2017.

[48] R. J. Plevin, A. D. Jones, M. S. Torn, and H. K. Gibbs, "Greenhouse gas emissions from biofuels' 682 indirect land use change are uncertain but may be much greater than previously estimated," Environ. 683 Sci. Technol., vol. 44, pp. 8015-8021, Nov. 2010.

[49] T. Searchinger, R. Heimlich, R. A. Houghton, F. Dong, A. Elobeid, J. Fabiosa, S. Tokgoz, D. Hayes, 685 and T.-H. Yu, "Use of u.s. croplands for biofuels increases greenhouse gases through emissions from 686 land-use change," Science, vol. 319, pp. 1238-1240, Feb. 2008.

[50] M. Fajardy, H. A. Daggash, and N. Mac Dowell, "Comparing BECCS and DAC for Climate Change 688 Mitigation: The Water-Land-Energy Nexus," in 2017 AIChE Annual Meeting, (Minneapolis, USA), 689 Nov. 2017.

[51] Office of Gas and Electricity Markets, "Renewables Obligation: Sustainability Criteria." 691 https://wWw.ofgem.gov.uk/system/files/docs/2016/03/ofgem_ro___ 692 sustainability_criteria_guidance_march_16.pdf, Mar. 2016.

[52] National Oceanic and Atmospheric Administration Earth System Research Laboratory, "Recent 694 Monthly Average Mauna Loa $\mathrm{CO}_{2}$." https: / /www.esrl.noaa.gov/gmd/ccgg/trends/, 695 Feb. 2018.

[53] K. S. Lackner, "Washing Carbon Out of the Air," Sci. Amer., vol. 302, pp. 66-71, June 2010.

[54] K. S. Lackner, “Capture of carbon dioxide from ambient air," The European Physical Journal Special 698 Topics, vol. 176, pp. 93-106, Sept. 2009.

[55] R. Socolow, M. Desmond, R. Aines, J. Blackstock, O. Bolland, T. Kaarsberg, N. Lewis, M. Mazzotti, 700 A. Pfeffer, K. Sawyer, J. Siirola, B. Smit, and J. Wilcox, "Direct Air Capture of $\mathrm{CO}_{2}$ with 701 Chemicals. A Technology Assessment for the APS Panel on Public Affairs." Available at: https: 702 //tinyurl.com/yd7rxssk, June 2011. 703

[ [56] Department of Energy \& Climate Change, “Updated energy and emissions projections 2015." http: 704 //tinyurl.com/n5a9yxv, 2015.

[57] C. F. Heuberger, I. Staffell, N. Shah, and N. Mac Dowell, "A systems approach to quantifying the 706 value of power generation and energy storage technologies in future electricity networks," Comput. 707 Chem. Eng., vol. 107, pp. 247-256, Dec. 2017.

[58] C. F. Heuberger, E. Rubin, I. Staffell, N. Shah, and N. Mac Dowell, "Power capacity expansion 709 planning considering endogenous technology cost learning," Appl. Energy, pp. 831-845, 2017.

[59] C. F. Heuberger, I. Staffell, N. Shah, and N. Mac Dowell, "Quantifying the value of ccs for the future 711 electricity system,” Energy Environ. Sci., vol. 9, no. 8, pp. 2497-2510, 2016.

[60] C. F. Heuberger, "Electricity Systems Optimisation with capacity eXpansion and Endogenous 713 technology Learning (ESO-XEL)." https://zenodo.org/record/1048943\# 714 .WleFma5l-Um, Nov. 2017.

[61] G. Appa, "The use of linear programming duality in mixed integer programming," IMA Journal of 716 Management Mathematics, vol. 8, pp. 225-242, Mar. 1997. 
[62] E. V. Pachkova, "Duality in mip by branch-and-cut: generating dual price functions using 718 branch-and-cut," Advanced Modeling and Optimization [electronic only], vol. 7, pp. 73-87, Jan. 719 2005.

[63] M. Guzelsoy and T. Ralphs, "Duality for mixed-integer linear programs," International Journal of 721 Operational Research, vol. 4, pp. 1-34, May 2007.

[64] M. Bui, M. Fajardy, and N. Mac Dowell, "Bio-energy with CCS (BECCS) performance evaluation: 723 Efficiency enhancement and emissions reduction," Applied Energy, vol. 195, pp. 289-302, June 2017.724

[65] M. Finkenrath, "Cost and Performance of Carbon Dioxide Capture from Power Generation." Working 725 Paper. Available at: https://tinyurl.com/y8grs8ee, 2011.

[66] Drax Group Ltd, "Half Year Results for the Six Months Ended 30 June 727 2015." https://www.drax.com/wp-content/uploads/2016/09/ 72 2015-Half-Year-Results-for-the-six-months-ended-30-June-20151.pdf, $\quad 729$ July 2015.

[67] EDINA Environment Digimap ${ }^{\circledR}$ Service, “Maps \& geospatial data for UK academia." http:// 731 digimap.edina.ac.uk/, 2017.

[68] Department for Environment, Food and Rural Affairs, Department of Agriculture, Environment and 733 Rural Affairs (Northern Ireland), Welsh Assembly Government, The Department for Rural Affairs 734 and Heritage, and The Scottish Government, Rural \& Environment Science \& Analytical Services, 735 “Agriculture In the United Kingdom 2016," May 2017.

[69] A. Hastings, M. J. Tallis, E. Casella, R. W. Matthews, P. A. Henshall, S. Milner, P. Smith, and 737 G. Taylor, "The technical potential of great britain to produce ligno-cellulosic biomass for bioenergy in current and future climates," GCB Bioenergy, vol. 6, pp. 108-122, Nov. 2013.

[70] Energy Technologies Institute, "Bioenergy crops in the UK: Case studies of successful whole farm 740 integration evidence pack." https://tinyurl.com/y7a34kwb, Oct. 2016.

[71] N. Seifkar, X. Lu, M. Withers, R. Malina, R. Field, S. Barrett, and H. Herzog, "Biomass to Liquid 742 Fuels Pathways: A Techno-Economic Environmental Evaluation." https://tinyurl.com/ 743 yahpsbn5, Mar. 2015.

[72] IEAGHG, "CO 2 Capture at Coal Based Power and Hydrogen Plants." https : / / inyurl.com/ jyw52w8, May 2014.

[73] D. Zhang, “Calculations.” Unpublished, Nov. 2017.

[74] Z. Wang, G. F. Weihs, P. Neal, and D. Wiley, "Effects of pipeline distance, injectivity and capacity on 748 $\mathrm{CO}_{2}$ pipeline and storage site selection," International Journal of Greenhouse Gas Control, vol. 51, 749 pp. 95-105, aug 2016.

[75] I. Staffell, “Electric Insights Quarterly." http://electricinsights.co.uk/\#/reports/ methodology?\&_k=1g6ifp, 2017.

[76] Office of Gas and Electricity Markets, "Electricity prices: Day-ahead baseload contracts - montly 753 average (GB)," Sept. 2017. https://www.ofgem.gov.uk/data-portal/all-charts\# 754 thumbchart-of_highcharts_block_view-n95196. 
[77] G. Ellis and G. Ferraro, "The social acceptance of wind energy: Where we stand and the path ahead." 756 Luxembourg: Publications office of the European Union (EUR 28182 EN), 2016.

[78] N. Mac Dowell and M. Fajardy, "Inefficient power generation as an optimal route to negative 758 emissions via BECCS?," Environmental Research Letters, vol. 12, p. 045004, Apr. 2017.

[79] Department for Business, Energy \& Industrial Strategy (BEIS), "Contracts for Difference 760 Second Allocation Round Results." https://www.gov.uk/government/uploads/ 761 system/uploads/attachment_data/file/643560/CFD_allocation_round_2_ 762 outcome_FINAL.pdf, Sept. 2017.

[80] M. Ha-Duong, A. Nadaï, and A. S. Campos, "A survey on the public perception of CCS in france," 764 Energy Procedia, vol. 1, pp. 4757-4764, Feb. 2009.

[81] K. Bickerstaff, G. Walker, and H. Bulkeley, Energy Justice in a Changing Climate. Zed Books Ltd, 766 2013.

[82] T. Adams and N. M. Dowell, "Off-design point modelling of a 420mw CCGT power plant integrated 768 with an amine-based post-combustion CO2 capture and compression process," Applied Energy, 769 vol. 178, pp. 681-702, Sept. 2016.

[83] S. Pye, F. G. N. Li, J. Price, and B. Fais, "Achieving net-zero emissions through the reframing of UK 771 national targets in the post-Paris Agreement era," Nature Energy, vol. 2, Mar. 2017.

[84] T. Boßmann and I. Staffell, "The shape of future electricity demand: Exploring load curves in 2050s 773 germany and britain," Energy, vol. 90, pp. 1317-1333, oct 2015.

q [85] National Grid Plc, "Data Explorer - Real Time Demand Data: Historic.” http://tinyurl.com/ kbe 65 ca, 2015.

[86] National Grid Plc, “System Operability Framework 2016.” https://tinyurl .com/guglwvy, 2016.

[87] P. Heptonstall, R. Gross, and F. Steiner, “The costs and impacts of intermittency âĂŞ 2016 update.” 779 https://tinyurl.com/y7bwbuo4, Feb. 2017.

[88] European Network of Transmission System Operators for Electricity, "Statistical Database." https : //www.entsoe.eu/, 2015.

[89] Department for Business, Energy \& Industrial Strategy, "Digest of United Kingdom Energy Statistics 783 2016." Available at: https://tinyurl.com/yaqn5pr3, 2016.

[90] National Grid Plc, "Special Condition 2K.4 - Transmission Losses Report: Reporting Period 1 April 785 2015 to 31 March 2016." https://tinyurl.com/y8zo54m6, 2016.

[91] Sohn Associates Limited, "Electricity Distribution Systems Losses: prepared for Office of Gas and Electricity Markets (Ofgem)." https://tinyurl.com/ joarop2, 2009.

[92] A. Flamm and D. Scott, "Electricity Balancing Significant Code Review - Final Policy Decision." 789 https://tinyurl.com/y7cwdlqc, May 2014.

[93] S. Pfenninger and I. Staffell, "Renewables ninja." https://www.renewables.ninja/, 2016. 791 
[94] I. Staffell and S. Pfenninger, "Using bias-corrected reanalysis to simulate current and future wind 792 power output," Energy, vol. 114, pp. 1224-1239, nov 2016.

[95] S. Pfenninger and I. Staffell, "Long-term patterns of european PV output using 30 years of validated 794 hourly reanalysis and satellite data," Energy, vol. 114, pp. 1251-1265, nov 2016.

口[96] World Nuclear Association, “The Economics of Nuclear Power." https://tinyurl.com/ h8lxckv, 2017.

[ [97] Department of Energy \& Climate Change, "Fossil fuel projections: 2015." https://tinyurl. 798 com/y9wxuktv, 2015.

[98] EDINA, “EDINA Digimap Service.”https://digimap.edina.ac.uk/, 2018.

口 [99] GEMCO Energy, "Wood pellet plant cost: A feasibility study of wood pellet plant." https:// tinyurl.com/y8ggkgfm, 2018.

[100] M. Mobini, T. Sowlati, and S. Sokhansanj, "A simulation model for the design and analysis of wood pellet supply chains," Applied Energy, vol. 111, pp. 1239-1249, nov 2013.

[101] Özlem Akgül, N. Mac Dowell, L. G. Papageorgiou, and N. Shah, "A mixed integer nonlinear 805 programming (MINLP) supply chain optimisation framework for carbon negative electricity 806 generation using biomass to energy with CCS (BECCS) in the UK," International Journal of 807 Greenhouse Gas Control, vol. 28, pp. 189-202, sep 2014.

[102] E. S. Rubin, I. M. Azevedo, P. Jaramillo, and S. Yeh, "A review of learning rates for electricity supply 809 technologies," Energy Policy, vol. 86, pp. 198-218, Nov. 2015.

[103] Department for Business, Energy \& Industrial Strategy, "Electricity Generation Costs." Available at: 811 https://tinyurl.com/y9q2zmrr, Nov. 2016.

[104] J. Chow, K. Ho, X. Du, H. S. Lee, and M. Pearson, "Experience from extensive two-shift operation of 680MW coal/gas-fired units at castle peak power station - Hong Kong," Operation, Maintenance and Material Issues, vol. 1, no. 2, 2001.

[105] A. Mullane, G. Bryans, and M. O’Malley, "Kinetic energy and frequency response comparison for 816 renewable generation systems.” International Conference on Future Power Systems, 2005.

[106] Mott MacDonald, “UK Electricity Generation Costs Update." Available at: https://tinyurl. 818 Com/nhldhmv, 2010.

[107] M. Finkenrath, "Cost and Performance of Carbon Dioxide Capture from Power Generation." https : 820 //tinyurl.com/yarvpwbf, 2011.

[108] IEAGHG, "Operating Flexibility of Power Plants with CCS, 2012/6." https://tinyurl.com/ 822 yb76wyo6, June 2012.

[109] D. Lew, G. Brinkman, E. Ibanez, A. Florita, M. Heaney, B. M. Hodge, M. Hummon, and G. Stark, 824 "The Western Wind and Solar Integration Study Phase 2." http://www.nrel.gov/docs/ 825 fy130sti/55588.pdf., 2013.

[110] R. Domenichini, L. Mancuso, N. Ferrari, and J. Davison, "Operating flexibility of power plants with 827 carbon capture and storage (CCS)," Energy Procedia, vol. 37, pp. 2727-2737, 2013. 
[111] Parsons Brinckerhoff, "Electricity Generation Model - 2013 Update Of NonRenewable Technologies: 829 for Department of Energy and Climate Change." Available at: https://tinyurl.com/ 830 oqdn 4 hd. 2013.

[112] R. Green, I. Staffell, and N. Vasilakos, "Divide and conquer? \$ $\{\mathrm{k}\}$ \$-means clustering of demand 832 data allows rapid and accurate simulations of the british electricity system," IEEE Transactions on 833 Engineering Management, vol. 61, pp. 251-260, May 2014.

[113] Parsons Brinckerhoff, "Technical Assessment of the Operation of Coal \& Gas Fired Plants." https : 835 //tinyurl.com/ybpa 7wus, Dec. 2014.

[114] Department of Engineering \& Public Policy, Carnergie Mellon University, "Integrated Environemntal 837 Control Model (IECM).”http://www.cmu.edu/epp/iecm/index.html, 2015.

[115] J. Morren, J. Pierik, and S. W. de Haan, "Inertial response of variable speed wind turbines," Electric Power Systems Research, vol. 76, pp. 980-987, July 2006.

[116] P. Tielens and D. van Hertem, "Grid Inertia and Frequency Control in Power Systems with High 84 Penetration of Renewables." https://tinyurl.com/h24h6uf, 2012.

[117] Department of Energy \& Climate Change, "Electricity Generation Costs 2013." https:// 843 tinyurl.com/o25or2g, 2013.

[118] N. Ceccarelli, M. van Leeuwen, T. Wolf, P. van Leeuwen, R. van der Vaart, W. Maas, and A. Ramos, 845 "Flexibility of low-CO2 gas power plants: Integration of the $\mathrm{CO} 2$ capture unit with CCGT operation," ${ }_{846}$ Energy Procedia, vol. 63, pp. 1703-1726, 2014.

[119] M. Nimtz and H.-J. Krautz, "Flexible operation of CCS power plants to match variable renewable energies," Energy Procedia, vol. 40, pp. 294-303, 2013.

[120] P. Versteeg, D. L. Oates, E. Hittinger, and E. S. Rubin, "Cycling coal and natural gas-fired power plants with CCS," Energy Procedia, vol. 37, pp. 2676-2683, 2013.

[121] International Energy Agency, "World Energy Investment Outlook: Special Report." https:// 852 tinyurl.com/yap49crx, 2014.

[122] N. Mac Dowell and M. Fajardy, "On the potential for BECCS efficiency improvement through heat 854 recovery from both post-combustion and oxy-combustion facilities," Faraday Discussions, vol. 192, 855 pp. 241-250, 2016.

[123] I. Tsiropoulos, D. Tarvydas, and A. Zucker, "Cost development of low carbon energy technologies - 857 Scenario-based cost trajectories to 2050, 2017 edition.” EUR 29034 EN, Publications Office of the 858 European Union, Luxembourg. Available at: https://tinyurl.com/y92c6soa, 2018. ISBN 859 978-92-79-77479-9.

[124] C. Moné, A. Smith, B. Maples, and M. Hand, "2013 Cost of Wind Energy Review." https:// 861 tinyurl.com/ybgtb3a7, Feb. 2015.

[125] M. Junginger, A. Faaij, and W. C. Turkenburg, "Cost reduction prospects for offshore wind farms," 863 Wind Engineering, vol. 28, pp. 97-118, Jan. 2004. 
[126] European Commission, "Electricity interconnection: Sweden - Lithuania (Nordbalt)." https:// 865 tinyurl.com/y7v2pvvo, 2013.

[127] MWH Americas Inc., "Technical Analysis of Pumped Storage and Integration with Wind Power in 867 the Pacific Northwest.” https: / / inyurl.com/ydeqqolj, 2009. page 166.

[128] B. B. McKeon, J. Furukawa, and S. Fenstermacher, "Advanced lead-acid batteries and the 869 development of grid-scale energy storage systems," Proceedings of the IEEE, vol. 102, pp. 951-963, 870 June 2014.

[129] S. Matteson and E. Williams, "Residual learning rates in lead-acid batteries: Effects on emerging 872 technologies," Energy Policy, vol. 85, pp. 71-79, Oct. 2015.

[130] U.S. Energy Information Administration, "Capital Cost Estimates for Utility Scale Electricity 874 Generating Plants.” Available at: https://tinyurl.com/y9tvv8zh, Nov. 2016.

[131] European Association for Storage of Energy and European Energy Research Alliance, "Joint 876 EASE/EERA Recommendation for a European Energy Storage Technology Development Roadmap 877 Towards 2030.” Available at: https://tinyurl.com/y98dbb9u, Oct. 2017. 


\section{Appendix 1}

The ESO-X model used in this work has been extended to include power-consuming negative emissions 880 technologies, e.g. DACS. The mathematical formulation of the model, described here ${ }^{58}$, has been adapted to include DACS. The equations changed are given below. Eq. 1-4 are newly added and are the basis of the analysis in this paper. The subsequent equations have been numbered to correspond with the labelling in the original model description ${ }^{58}$. An accessible version of the ESO-X model is available ${ }^{60}$.

Table 2: Sets, parameters and variables added/updated in the ESO-X model

\begin{tabular}{|c|c|c|c|}
\hline Type & & Description & Unit \\
\hline \multirow{7}{*}{ Set } & $i$ & all technologies considered in the system & - \\
\hline & $i g$ & power generating technologies, ig $\subseteq i$ & - \\
\hline & is & storage technologies, is $\subseteq i$ & - \\
\hline & $t$ & time & hours \\
\hline & $a$ & planning period & years \\
\hline & $c$ & cluster set obtained using k-means clustering & - \\
\hline & $n e$ & negative emissions technologies, $n e \subset i$ & - \\
\hline \multirow{23}{*}{ Parameter } & $W F_{c}$ & weighting factor for cluster $c$ & - \\
\hline & $\operatorname{Disc}_{a}$ & discount factor in year $a$ & - \\
\hline & $\operatorname{Des}_{i}$ & unit capacity of technology $i$ & MW/unit \\
\hline & $\operatorname{Pmin}_{i}$ & minimum power output from technology $i$ & $\%-\mathrm{MW}$ \\
\hline & $\operatorname{Pmax}_{i}$ & maximum power output from technology $i$ & $\%-\mathrm{MW}$ \\
\hline & $C A P E X_{i}$ & capital cost of tecnology $i$ & $£ /$ unit \\
\hline & $E m s_{i}$ & carbon emissions from technology $i$ & $\mathrm{t}_{\mathrm{CO}_{2}} / \mathrm{MWh}$ \\
\hline & $O P E X_{i, a}$ & operational cost of technology $i$ & $£ / \mathrm{MWh}$ \\
\hline & $O P E X S U_{i g}$ & start-up costs of technology ig & $£$ \\
\hline & $O P E X N L_{i}$ & fixed operational costs of technology $i$ & $£ / \mathrm{MWh}$ \\
\hline & SEta & storage roundtrip efficiency & $\%$ \\
\hline & $S E_{a}$ & annual system carbon emissions & $\mathrm{t}_{\mathrm{CO}_{2}}$ \\
\hline & ImpElecPr $r_{t}$ & import electricity price at time $t$ of cluster $c$ & $£ / \mathrm{MWh}$ \\
\hline & VoLL & value of lost load & $£ / \mathrm{MWh}$ \\
\hline & $U K N E T_{a}$ & UK negative emissions target in year $a$ & $\mathrm{t}_{\mathrm{CO}_{2}}$ \\
\hline & $b_{i, a}$ & number of units of technology $i$ built in year $a$ & - \\
\hline & $d_{i, a}$ & number of units of technology $i$ installed in year $a$ & - \\
\hline & $u_{i g, a, c, t}$ & $\begin{array}{l}\text { number of units of technology ig starting up at time } t \text { in } \\
\text { year } a \text { of cluster } c\end{array}$ & - \\
\hline & nets $_{n e, a, c, t}$ & $\begin{array}{l}\text { number of units of DACS units } n e \text { operating at time } t \text { in } \\
\text { year } a \text { of cluster } c\end{array}$ & - \\
\hline & $p_{i g, a, c, t}$ & $\begin{array}{l}\text { energy output of technology ig to demand at time } t \text { in year } \\
a \text { of cluster } c\end{array}$ & MWh \\
\hline & $p 2 d_{i g, a, c, t}$ & $\begin{array}{l}\text { energy from technology } i g \text { to demand at time } t \text { in year } a \text { of } \\
\text { cluster } c\end{array}$ & MWh \\
\hline & $p 2 s_{i g, a, c, t}$ & $\begin{array}{l}\text { energy from technology ig to storage at time } t \text { in year } a \text { of } \\
\text { cluster } c\end{array}$ & MWh \\
\hline & $p 2 D A C S_{i g, a, c, t}$ & $\begin{array}{l}\text { energy from generating technology ig to DACS at time } t \text { in } \\
\text { year } a \text { of cluster } c\end{array}$ & MWh \\
\hline
\end{tabular}


Table 2: Sets, parameters and variables added/updated in the ESO-X model

\begin{tabular}{|c|c|c|c|}
\hline \multirow[t]{2}{*}{ Type } & & Description & Unit \\
\hline & $p 2 i s_{i s, a, c, t}$ & $\begin{array}{l}\text { energy to storage technology is at time } t \text { in year } a \text { of cluster } \\
c\end{array}$ & MWh \\
\hline \multirow[t]{9}{*}{ Variable } & $s_{i s, a, c, t}$ & $\begin{array}{l}\text { effective state of charge of technology is at the end of time } \\
t \text { in year } a \text { of cluster } c\end{array}$ & MWh \\
\hline & $s 2 d_{i s, a, c, t}$ & $\begin{array}{l}\text { energy from storage technology is to demand at time } t \text { in } \\
\text { year } a \text { of cluster } c\end{array}$ & MWh \\
\hline & $s 2 r_{i s, a, c, t}$ & $\begin{array}{l}\text { energy from storage technology } i s \text { to reserve at time } t \text { in } \\
\text { year } a \text { of cluster } c\end{array}$ & MWh \\
\hline & $s 2 D A C S_{i s, a, c, t}$ & $\begin{array}{l}\text { energy from storage technology is to DACS at time } t \text { in } \\
\text { year } a \text { of cluster } c\end{array}$ & MWh \\
\hline & $s l a k_{a, c, t}$ & unmet electricity demand at time $t$ in year $a$ of cluster $c$ & MWh \\
\hline & $D A C S O P E X$ & total operational costs of DACS technology & $£$ \\
\hline & $N E D A C S_{n e, a, c, t}$ & $\begin{array}{l}\text { negative emissions provided by DACS at time } t \text { in year } a \\
\text { of cluster } c\end{array}$ & $\mathrm{t}_{\mathrm{CO}_{2}}$ \\
\hline & $e_{i g, a, c, t}$ & $\begin{array}{l}\text { carbon emissions from technology ig at time } t \text { in year } a \text { of } \\
\text { cluster } c\end{array}$ & $\mathrm{t}_{\mathrm{CO}_{2}}$ \\
\hline & $t s e_{a}$ & $\begin{array}{l}\text { total system carbon emissions in year } a \\
\text { total system cost }\end{array}$ & $\begin{array}{l}\mathrm{t}_{\mathrm{CO}_{2}} \\
\mathrm{f}\end{array}$ \\
\hline
\end{tabular}

\section{Equations}

$$
\begin{aligned}
& n e t s_{n e, a, c, t} \leq d_{n e, a} \quad \forall n e, a, c, t \\
& \sum_{i g} p 2 D A C S_{i g, a, c, t}+\sum_{i s} s 2 D A C S_{i s, a, c, t} \leq n e t s_{n e, a, c, t} \text { Des }_{n e} \text { Pmax }_{n e} \quad \forall n e, a, c, t \\
& \sum_{i g} p 2 D A C S_{i g, a, c, t}+\sum_{i s} s 2 D A C S_{i s, a, c, t} \geq n e t s_{n e, a, c, t} \operatorname{Des}_{n e} \operatorname{Pmin}_{n e} \quad \forall n e, a, c, t \\
& N E D A C S_{n e, a, c, t}=\left(\sum_{i g} p 2 D A C S_{i g, a, c, t}+\sum_{i s} s 2 D A C S_{i s, a, c, t}\right) E m s_{n e} \quad \forall n e, a, c, t \\
& \sum_{i g, c, t} e_{i g, a, c, t} W F_{c}+\sum_{n e, c, t} N E D A C S_{n e, a, c, t} W F_{c} \leq S E_{a}+U K N E T_{a} \quad \forall a \\
& p 2 d_{i g, a, c, t}+p 2 s_{i g, a, c, t}+p 2 D A C S_{i g, a, c, t}=p_{i g, a, c, t} \quad \forall i g, a, c, t \\
& s 2 d_{i s, a, c, t}+s 2 r_{i s, a, c, t}+s 2 D A C S_{i s, a, c, t} \geq o_{i s, a, c, t} \operatorname{Des}_{i s} \operatorname{Pmin}_{i s} \quad \forall i s, a, c, t \\
& s 2 d_{i s, a, c, t}+s 2 r_{i s, a, c, t}+s 2 D A C S_{i s, a, c, t} \leq o_{i s, a, c, t} \text { Des }_{i s} \quad \forall i s, a, c, t
\end{aligned}
$$




$$
\begin{aligned}
& s 2 d_{i s, a, c, t}+s 2 r_{i s, a, c, t}+s 2 D A C S_{i s, a, c, t} \leq s_{i s, a, c, t} S E t a_{i s} \quad \forall i s, a, c, t \\
& s_{i s, a, c, t}=s_{i s, a, c, t-1}-s 2 d_{i s, a, c, t}-s 2 D A C S_{i s, a, c, t}+p 2 i s_{i s, a, c, t} S E t a_{i s} \quad \forall i s, a, c, t>1 \\
& t s c=\sum_{i} C A P E X_{i} b_{i, a} \text { Des }_{i} / \operatorname{Disc}_{a}+\sum_{i g, a, c, t}\left(u_{i g, a, c, t} O P E X S U_{i g} W F_{c}\right) / D_{i s c_{a}} \\
& +\sum_{i g, a, c, t}\left(O P E X_{i g, a} p_{i g, a, c, t} W F_{c}+O P E X N L_{i g} n_{i g, a, c, t} W F_{c}\right) / D i s c_{a} \\
& +\sum_{i s, a, t}\left(O P E X_{i s, a}\left(s 2 d_{i g, a, c, t}+s 2 D A C S_{i s, a, c, t}\right) W F_{c}+O P E X N L_{i s} o_{i s, a, c, t} W F_{c}\right) / D i s c_{a} \\
& +\sum_{i=\text { InterImp }, a, t} \operatorname{ImpElecPr}_{t} p 2 d_{i, a, c, t} W F_{c} / \operatorname{Disc}_{a}+\sum_{a, c, t} \operatorname{slak}_{a, c, t} W F_{c} \operatorname{VoLL} \\
& +\sum_{n e, a} O P E X_{n e, a}\left(\sum_{i g, c, t} p 2 D A C S_{i g, a, c, t} W F_{c}+\sum_{i s} s 2 D A C S_{i s, a, c, t} W F_{c}\right) / D i s c_{a}
\end{aligned}
$$




\section{Appendix 2}

\section{System parameters}

The base year for the ESO-X model is 2015. The annual demand and other system parameters are as 888 provided in Table 3. We assume a $1 \%$ year-on-year increase in electricity demand. Although demand in 889 the UK has fallen in recent years, increasing electrification of other sectors of the economy (heating and 890 transport) is expected to result in rising demand 83184 .

Table 3: Data sources for system parameters assumed in the ESO-XEL model.

\begin{tabular}{|c|c|}
\hline UK electricity system & Data source(s) \\
\hline Hourly electricity demand & 85 \\
\hline System inertia requirement & 86 \\
\hline Capacity reserve for intermittent renewables & 87 \\
\hline $\mathrm{CO}_{2}$ emissions targets until 2050 & 56 \\
\hline Price of imported electricity & 88 \\
\hline Power transmission and distribution losses & $89-91$ \\
\hline Value of lost load & 92 \\
\hline Hourly availability of solar, onshore wind and offshore wind & $93-95$ \\
\hline Carbon price & 17 \\
\hline $\mathrm{CO}_{2}$ transport and storage costs & 72174 \\
\hline
\end{tabular}

\section{Fuel prices}

Table 4: Data sources for fuel costs assumed in the ESO-X model.

\begin{tabular}{|ll|}
\hline Fuel cost & Data sources \\
\hline Uranium $\left(\mathrm{UO}_{2}\right.$ fuel $)$ & {$[96$} \\
Coal & {$[97$} \\
Natural gas & 97 \\
\hline
\end{tabular}

\section{Biomass supply chain}

The cost of biomass fuel is implemented using a supply chain. Two sources of biomass are considered: 894 indigenous virgin biomass (Miscanthus is the grass considered) and pellet imports. For the local biomass, 895 full supply chain (harvest, processing and pelletisation) emissions and costs are considered. For the imported 896 biomass, transport costs from UK ports are considered. Tables 5 - 7 detail the costs and associated $\mathrm{CO}_{2} \quad 897$ emissions of the biomass supply chain. 
Table 5: Biomass supply and processing costs. Land availability is obtained from 98 .

\begin{tabular}{|c|c|c|c|c|}
\hline Biomass type & $\begin{array}{l}\text { Raw material } \\
\text { (£/tonne) }\end{array}$ & $\begin{array}{l}\text { Pellet conversion } \\
\text { rate }\end{array}$ & $\begin{array}{l}\text { Pellet production } \\
\text { cost ( } £ / \text { tonne) }\end{array}$ & $\begin{array}{l}\text { Availability } \\
\text { (tonne/ha yr) }\end{array}$ \\
\hline Miscanthus & 4970 & $83.7 \% 99$ & $17.86^{100}$ & 69 \\
\hline Imported pellets & $159.5^{71}$ & - & - & \\
\hline
\end{tabular}

Table 6: Cost of pellet plant

\begin{tabular}{ll}
\hline Pellet plant & \\
\hline Unit capacity (tonnes/hr) & $20 \sqrt{100}$ \\
Capital costs (M£/unit) & $12 \sqrt{100}$ \\
Lifetime (years) & $30^{101}$ \\
\hline
\end{tabular}

Technology parameters 
Table 7: Transportation costs and emissions assumed.

\begin{tabular}{lll}
\hline Transportation & $\begin{array}{l}\text { Unit transport cost } \\
(\mathfrak{f} / \mathrm{t} \mathrm{km})^{101}\end{array}$ & $\begin{array}{l}\text { Unit emissions } \\
\left(\mathrm{kg}_{\mathrm{CO}_{2}} / \mathrm{t} \mathrm{km}\right)^{101}\end{array}$ \\
\hline Truck & 0.47 & 0.062 \\
Rail & 0.17 & 0.022 \\
Ship & 0.06 & 0.005 \\
\hline
\end{tabular}

Table 8: Data sources for the technology costs and operational parameters implemented in the ESO-X model. Capital costs (average) and efficiencies are highlighted.

\begin{tabular}{|c|c|c|c|}
\hline Technology & $\begin{array}{l}\text { CAPEX } \\
(£ / k W)\end{array}$ & $\begin{array}{l}\text { Efficiency } \\
\text { (HHV) }\end{array}$ & $\begin{array}{l}\text { Data sources } \\
\text { reviewed }\end{array}$ \\
\hline Nuclear & 3673 & $37.0 \%$ & 102103 \\
\hline Coal (super-critical) & 1237 & $42.2 \%$ & $72 \quad 102 \quad 104-114$ \\
\hline Dedicated biomass-fired & 1590 & $40.0 \%$ & 103 \\
\hline Combined cycle gas turbine (CCGT) & 540 & $52.7 \%$ & 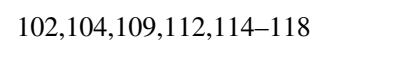 \\
\hline Open cycle gas turbine (OCGT) & 459 & $39.0 \%$ & $102 \quad 104 / 06 \quad 109: 113-117$ \\
\hline Coal post-combustion CCS & 2368 & $33.6 \%$ & 72/105-107/110,114]117]|119-121 \\
\hline CCGT post-combustion CCS & 1187 & $45.5 \%$ & \begin{tabular}{|l|lllll|l|l|}
102 & 107 & 108 & 110 & 117 & 120 & 121 \\
\end{tabular} \\
\hline Bioenergy with CCS (BECCS) & 2721 & $35.0 \%$ & $476472 \quad 121-123$ \\
\hline Onshore Wind & 1105 & - & $93 / 102 / 103 / 115-117,124$ \\
\hline Offshore Wind & 2600 & - & $93 / 102 / 03 / 15-117,124$ \\
\hline Solar Photovoltaic (PV) & 1157 & - & 93102 \\
\hline High-voltage direct current interconnection (HVDC) & 1000 & $52 \%$ & 117125126 \\
\hline Pumped hydro & 1875 & - & 102121127 \\
\hline Lead-acid battery & 1400 & - & 128-131 \\
\hline Direct air capture and storage (DACS) & 33074 & - & 55 \\
\hline
\end{tabular}

